\title{
Distributed Computing With the Cloud
}

\author{
Yehuda Afek Gal Giladi Boaz Patt-Shamir \\ School of CS School of EE \\ Tel Aviv University \\ Tel Aviv 6997801 \\ Israel
}

September 28, 2021

\begin{abstract}
We investigate the effect of omnipresent cloud storage on distributed computing. We specify a network model with links of prescribed bandwidth that connect standard processing nodes, and, in addition, passive storage nodes. Each passive node represents a cloud storage system, such as Dropbox, Google Drive etc. We study a few tasks in this model, assuming a single cloud node connected to all other nodes, which are connected to each other arbitrarily. We give implementations for basic tasks of collaboratively writing to and reading from the cloud, and for more advanced applications such as matrix multiplication and federated learning. Our results show that utilizing node-cloud links as well as node-node links can considerably speed up computations, compared to the case where processors communicate either only through the cloud or only through the network links.

We provide results for general directed graphs, and for graphs with "fat" links between processing nodes. For the general case, we provide optimal algorithms for uploading and downloading files using flow techniques. We use these primitives to derive algorithms for combining, where every processor node has an input value and the task is to compute a combined value under some given associative operator. In the case of fat links, we assume that links between processors are bidirectional and have high bandwidth, and we give near-optimal algorithms for any commutative combining operator (such as vector addition). For the task of matrix multiplication (or other noncommutative combining operators), where the inputs are ordered, we present sharp results in the simple "wheel" network, where procesing nodes are arranged in a ring, and are all connected to a single cloud node.
\end{abstract}

\section{Introduction}

In 2018 Google announced that the number of users of Google Drive is surpassing one billion [25]. Earlier that year, Dropbox stated that in total, more than an exabyte ( $10^{18}$ bytes) of data has been uploaded by its users [14]. Other cloud-storage services, such as Microsoft's OneDrive, Amazon's S3, or Box, are thriving too. The driving force of this paper is our wish to let other distributed systems to take advantage of the enormous infrastructure that makes up the complexes called "clouds." Let us explain how. 


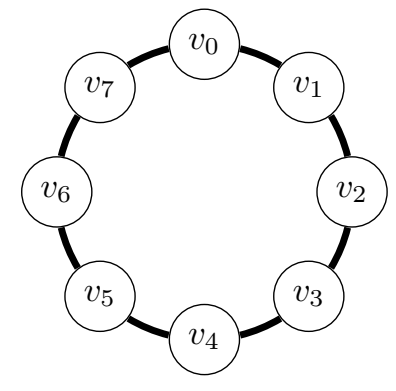

Local edges only

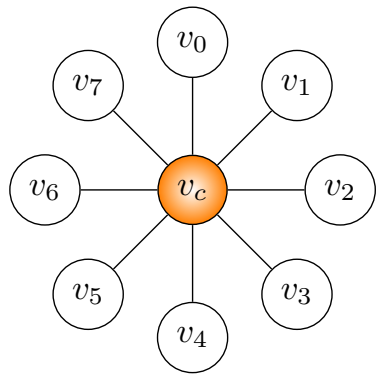

Cloud edges only

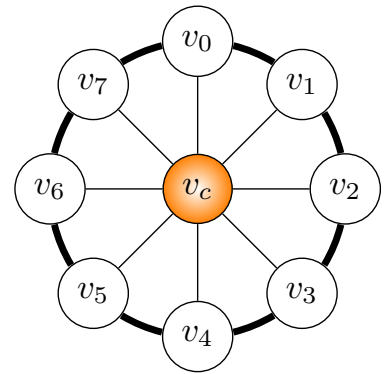

Computing with the cloud

Figure 1: Wheel topology with $n=8$. The $v_{i}$ nodes are processing nodes connected by a ring of highbandwidth links. The cloud node $v_{c}$ is connected to the processing nodes by lower-bandwidth links. All links are bidirectional and symmetric.

The computational and storage capacities of servers in cloud services are relatively well advertised. A lesser known fact is that a cloud system also entails a massive component of communication, that makes it appear close almost everywhere on the Internet. (This feature is particularly essential for cloud-based video conferencing applications, such as Zoom, Cisco's Webex and others.) In view of the existing cloud services, our fundamental idea is to abstract a complete cloud system as a single, passive storage node.

To see the benefit of this appraoch, consider a network of the "wheel" topology: a single cloud node is connected to $n$ processing nodes arranged in a cycle (see Fig. 1). Suppose each processing node has a wide link of bandwidth $n$ to its cycle neighbors, and a narrower link of bandwidth $\sqrt{n}$ to the cloud node. Further suppose that each processing node has an $n$-bit vector, and that the goal is to calculate the sum of all vectors. Without the cloud (Fig. 1, left), such a task requires at least $\Omega(n)$ rounds - to cover the distance; on the other hand, without using the cycle links (Fig. 1, middle), transmitting a single vector from any processing node (and hence computing the sum) requires $\Omega(n / \sqrt{n})=\Omega(\sqrt{n})$ rounds - due to the limited bandwidth to the cloud. But using both cloud links and local links (Fig. 1, right), the sum can be computed in $\tilde{\Theta}(\sqrt[4]{n})$ rounds, as we show in this paper.

More generally, in this paper we initiate the study of the question of how to use an omnipresent cloud storage to speed up computations, if possible. We stress that the idea here is to develop a framework and tools that facilitate computing with the cloud, as opposed to computing in the cloud.

Specifically, in this paper we introduce the computing with the cloud model (CWC), and present algorithms that efficiently combine distributed inputs to compute various functions, such as vector addition and matrix multiplication. To this end, we first implement (using dynamic flow techniques) primitive operations that allow for the exchange of large messages between processing nodes and cloud nodes. Given the combining algorithms, we show how to implement some applications such as federated learning and file de-duplication (dedup). 


\subsection{Model Specification}

The "Computing with the Cloud" (CWC) model is a synchronous network whose underlying topology is described by a weighted directed graph $G=(V, E, w)$. The node set consists of two disjoint subsets: $V=V_{p} \cup V_{c}$, where $V_{p}$ is the set of processing nodes, and $V_{c}$ is the set of cloud nodes. Cloud nodes are passive nodes that function as shared storage: they support read and write requests, and do not perform any other computation. We use $n$ to denote the number of processing nodes (the number of cloud nodes is typically constant).

We denote the set of links that connect two processing nodes by $E_{L}$ ("local links"), and by $E_{C}$ ("cloud links") the set of links that connect processing nodes to cloud nodes. Each link $e \in E=E_{L} \cup E_{C}$ has a prescribed bandwidth $w(e)$ (there are no links between different cloud nodes). We denote by $G_{p} \stackrel{\text { def }}{=}\left(V_{p}, E_{L}\right)$ the graph $G-V_{c}$, i.e., the graph spanned by the processing nodes.

Our execution model is the standard synchronous network model, where each round consists of processing nodes receiving messages sent in the previous round, doing an arbitrary local computation, and then sending messages. The size of a message sent over a link $e$ in a round is at most $w(e)$ bits.

Cloud nodes do not perform any computations: they can only receive requests we denote by FR and FW (file read and write, respectively), to which they respond in the following round. More precisely, each cloud node has unbounded storage; to write, a processing node $v_{i}$ invokes FW with arguments that describe the target cloud node, a filename $f$, a bit string $S$, and the location (index) within $f$ that $S$ needs to be written in. It is assumed that $|S| \leq w\left(v_{i}, v_{c}\right)$ bits (longer writes can be broken to a series of FW operations). To read, a processing node $v_{i}$ invokes FR with arguments that describe the cloud node, a filename $f$ and the range of indices to fetch from $f$. Again, we assume that the size of the range in any single $\mathrm{FR}$ invocation by node $v_{i}$ is at most $w\left(v_{i}, v_{c}\right){ }^{1}$

FW operations are exclusive, i.e., no other operation (read or write) to the same file location is allowed to take place simultaneously. Concurrent FR operations from the same location are allowed.

Discussion. We believe that our model is fairly widely applicable. A processing node in our model may represent anything from a computer cluster with a single gateway to the Internet, to cellphones or even smaller devices - anything with a non-shared Internet connection. The local links can range from high-speed fiber to Bluetooth or infrared links. Typically in this setting the local links have bandwidth much larger than the cloud links (and cloud downlinks in many cases have larger bandwidth than cloud uplinks). Another possible interpretation of the model is a private network (say, in a corporation), where a cloud node represents a storage or a file server. In this case the cloud link bandwidth may be as large as the local link bandwidth.

\footnotetext{
${ }^{1}$ For both the FW and FR operations we ignore the metadata (i.e., $v_{c}$ 's descriptor, the filename $f$ and the indices) and assume that the total size of metadata in a single round is negligible and can fit within $w\left(v_{i}, v_{c}\right)$. Otherwise, processing nodes may use the metadata parameters to exchange information that exceeds the bandwidth limitations (for example, naming a file with the string representation of a message whose length is larger than the bandwidth).
} 


\subsection{Problems Considered and Main Results}

Our main results in this paper are efficient algorithms in the CWC model to combine values stored at nodes. These algorithms use building blocks that facilitate efficient transmission of large messages between processing nodes and cloud nodes. These building blocks, in turn, are implemented in a straightforward way using dynamic flow techniques. Finally, we show how to use the combining algorithms to derive new algorithms for federated learning and file de-duplication (dedup) in the CWC model. More specifically, we provide implementations of the following tasks.

Basic cloud operations: Let $v_{c}$ denote a cloud node below.

- $c W_{i}$ (cloud write): write an $s$-bits file $f$ stored at node $i \in V_{p}$ to node $v_{c}$.

- $c R_{i}$ (cloud read): fetch an $s$-bits file $f$ from node $v_{c}$ to node $i \in V_{p}$.

- cAW (cloud all write): for each $i \in V_{p}$, write an $s$-bits file $f_{i}$ stored at node $i$ to node $v_{c}$.

- cAR (cloud all read): for each $i \in V_{p}$, fetch an $s$-bits file $f_{i}$ from node $v_{c}$ to node $i$.

Combining and dissemination operations:

- cComb: (cloud combine): Each node $i \in V_{p}$ has an $s$-bits input string $S_{i}$, and there is a binary associative operator $\otimes:\{0,1\}^{s} \times\{0,1\}^{s} \rightarrow\{0,1\}^{s}$ (the result is as long as each operand). The requirement is to write to a cloud node $v_{c}$ the $s$-bits string $S_{1} \otimes S_{2} \otimes \cdots \otimes S_{n}$. Borrowing from Group Theory, we call the operation $\otimes$ multiplication, and $S_{1} \otimes S_{2}$ is the product of $S_{1}$ by $S_{2}$. In general, $\otimes$ is not necessarily commutative. We assume the existence of a unit element for $\otimes$, denoted $\tilde{\mathbf{1}}$, such that $\tilde{\mathbf{1}} \otimes S=S \otimes \tilde{\mathbf{1}}=S$ for any $s$-bits strings $S$. The unit element is represented by a string of $O(1)$ bits. Examples for commutative operators include vector (or matrix) addition over a finite field, logical bitwise operations, leader election, and the top- $k$ problem. Examples for non-commutative operators may be matrix multiplication (over a finite field) and function composition.

- cCast (cloudcast): All the nodes $i \in V_{p}$ simultaneously fetch a copy of an $s$-bits file $f$ from node $v_{c}$. (Similar to network broadcast.)

Applications. cComb and cCast can be used directly to provide matrix multiplication, matrix addition, and vector addition. We also outline the implementation of the following.

Federated learning (FL) [31]: In FL, a collection of agents collaborate in training a neural network to construct a model of some concept, but the agents want to keep their data private. Unlike [31], in our model the central server is a passive storage device that does not carry out computations. We show how elementary secure computation techniques, along with our combining algorithm, can efficiently help training an ML model in the federated scheme implemented in CWC, while maintaining privacy. File deduplication: Deduplication (or dedup) is a task in file stores, where redundant identical copies of data are identified (and possibly unified) - see, e.g., [32]. Using cComb and cCast, we implement file dedup in the CWC model on collections of files stored at the different processing nodes. The algorithm keeps a single copy of each file and pointers instead of the other replicas.

Special topologies. The complexity of the general algorithms we present depends on the given network topology. We study a few cases of interest. 
First, we consider $s$-fat-links network, defined to be, for a given parameter $s \in \mathbb{N}$, as the CWC model with the following additional assumptions:

- All links are symmetric, i.e., $w(u, v)=w(v, u)$ for every link $(u, v) \in E$.

- Local links have bandwidth at least $s$.

- There is only one cloud node $v_{c}$.

The fat links model seems suitable in many real-life cases where local links are much wider than cloud links (uplinks to the Internet), as is the intuition behind the HYBRID model [4].

Another topology we consider is the wheel network, depicted schematically in Fig. 1 (right). In a wheel system there are $n$ processing nodes arranged in a ring, and a cloud node connected to all processing nodes. In the uniform wheel, all cloud links have the same bandwidth $b_{c}$ and all local links have the same bandwidth $b_{l}$. In the uniform wheel model, we typically assume that $b_{c} \ll b_{\ell}$.

The wheel network is motivated by non-commutative combining operations, where the order of the operands induces a linear order on the processing nodes, i.e., we view the nodes as a line, where the first node holds the first input, the second node holds the second input etc. For symmetry, we connect the first and the last node, and with a cloud node connected to all — we've obtained the wheel.

Overview of techniques. As mentioned above, the basic file operations (cW, cR, cAW and cAR) are solved optimally using dynamic flow techniques, or more specifically, quickest flow (Sec. 2, which have been studied in numerous papers in the past (cf. [9, 34]). We present closed-form bounds on cW and $\mathrm{cR}$ for the wheel topology in Sec. 4.

We present tight bounds for $\mathrm{cW}$ and $\mathrm{cR}$ in the $s$-fat-links network, where $s$ is the input size at all nodes. We then continue to consider the tasks cComb with commutative operators and cCast, and prove nearly-tight bounds on their time complexity in the $s$-fat-links network (Theorem 3.15, Theorem 3.17, Theorem 3.19). The idea is to first find, for every processing node $i$, a cluster of processing nodes that allows it to perform $\mathrm{cW}$ in an optimal number of rounds. We then perform cComb by combining the values within every cluster using convergecast [33], and then combining the results in a computation-tree fashion. Using sparse covers [5], we perform the described procedure in near-optimal time.

Non-commutative operators are explored in the natural wheel topology. We present algorithms for wheel networks with arbitrary bandwidth (both cloud and local links). We prove an upper bound for cComb (Theorem 4.6 ) and a lower bound of (Theorem 4.10).

Finally, in Sec. 5, we demonstrate how the considered tasks can be applied for the purposes of Federated Learning and File Deduplication.

Paper organization. In Sec. 2 we study the topology of basic primitives in the CWC model. In Sec. 3 we study combining algorithms in general topologies in fat links networks. In Sec. 4 we consider combining for non-commutative operators in the wheel topology. In Sec. 5 we discuss application level usage of the CWC model, such as federated learning and deduplication. Conclusions and open problems are presented in Sec. 6. 


\subsection{Related Work}

Our model is based on, and inspired by, a long history of theoretical models in distributed computing. To gain some perspective, we offer here a brief review.

Historically, distributed computing is split along the dichotomy of message passing vs shared memory [16]. While message passing is deemed the "right" model for network algorithms, the shared memory model is the abstraction of choice for programming multi-core machines.

The prominent message-passing models are LOCAL [28], and its derived CONGEST [33]. (Some models also include a broadcast channel, e.g. [2].) In both LOCAL and CONGEST, a system is represented by a connected (typically undirected) graph, in which nodes represent processors and edges represent communication links. In LOCAL, message size is unbounded, while in CONGEST, message size is restricted, typically to $O(\log n)$ bits. Thus, CONGEST accounts not only for the distance information has to traverse, but also for information volume and the bandwidth available for its transportation.

While most algorithms in the LOCAL and CONGEST models assume fault-free (and hence synchronous) executions, in the distributed shared memory model, asynchrony and faults are the primary source of difficulty. Usually, in the shared memory model one assumes that there is a collection of "registers," accessible by multiple threads of computation that run at different speeds and may suffer crash or even Byzantine faults (see, e.g., [3]). The main issues in this model are coordination and fault-tolerance. Typically, the only quantitative hint to communication cost is the number and size of the shared registers.

Quite a few papers consider the combination of message passing and shared memory, e.g., [30, 18, $12,19,35,1]$. The uniqueness of the CWC model with respect to past work is that it combines passive storage nodes with a message passing network with restrictions on the links bandwidth.

The CONGESTED CLIQUE (CC) model [29] is a special case of CONGEST, where the underlying graph is assumed to be fully connected. The CC model is appropriate for computing in the cloud, as it has been shown that under some relatively mild conditions, algorithms designed for the CC model can be implemented in the MapReduce model, i.e., run in datacenters [20]. Another model for computing in the cloud is the MPC model [22]. Very recently, the HYBRID model [4] was proposed as a combination of CC with classical graph-based communication. More specifically, the HYBRID model assumes the existence of two communication networks: one for local communication between neighbors, where links are typically of infinite bandwidth (exactly like LOCAL); the other network is a node-congested clique, i.e., a node can communicate with every other node directly via "global links," but there is a small upper bound (typically $O(\log n)$ ) on the total number of messages a node can send or receive via these global links in a round. Even though the model was presented only recently, there is already a line of algorithmic work in it, in particular for computing shortest paths [4, 23, 10].

Discussion. Intuitively, our CWC model can be viewed as the classical CONGEST model over the processors, augmented by special cloud nodes (object stores) connected to some (typically, many) compute nodes. To reflect modern demands and availability of resources, we relax the very stringent bandwidth allowance of CONGEST, and usually envision networks with much larger link bandwidth (e.g., $n^{\epsilon}$ for some $\epsilon>0$ ). 
Considering previous network models, it appears that HYBRID is the closest to CWC, even though HYBRID was not expressly designed to model the cloud. In our view, CWC is indeed more appropriate for computation with the cloud. First, in most cases, global communication (modeled by clique edges in HYBRID) is limited by link bandwidth, unlike HYBRID's node capacity constraint, which seems somewhat artificial. Second, HYBRID is not readily amenable to model multiple clouds, while this is a natural property of CWC.

Regarding shared memory models, we are unaware of topology-based bandwidth restriction on shared memory access in distributed models. In some general-purpose parallel computation models (based on BSP [35]), communication capabilities are specified using a few global parameters such as latency and throughput, but these models deliberately abstract topology away. In distributed (asynchronous) shared memory, the number of bits that need to be transferred to and from the shared memory is seldom explicitly analyzed.

\section{Implementation of Basic Communication Primitives in CWC}

In this section we give tight complexity results for the basic operations of reading or writing to the cloud, by one or all processors. The results are derived using standard dynamic flow techniques. We first review dynamic flows in Sec. 2.1, and then apply them to the CWC model in Sec. 2.2.

\subsection{Dynamic Flows}

The concept of quickest flow [9], a variant of dynamic flow [34], is defined as follows. ${ }^{2}$ A flow network consists of a directed weighted graph $G=(V, E, c)$ where $c: E \rightarrow \mathbb{N}$, with a distinguished source and sink nodes, denoted $s, t \in V$, respectively. A dynamic flow with time horizon $T \in \mathbb{N}$ and flow value $F$ is a mapping $f: E \times[1, T] \rightarrow \mathbb{N}$ that specifies for each edge $e$ and time step $j$, how much flow $e$ carries between steps $j-1$ and $j$, subject to the natural constraints:

- Edge capacities. For all $e \in E, j \in[1, T]$ :

$$
f(e, j) \leq c(e)
$$

- Only arriving flow can leave. For all $v \in V \backslash\{s\}, j \in[1, T-1]$ :

$$
\sum_{i=1}^{j} \sum_{(u, v) \in E} f((u, v), i) \geq \sum_{i=1}^{j+1} \sum_{(v, w) \in E} f((v, w), i)
$$

- No leftover flow. For all $v \in V \backslash\{s, t\}$ :

$$
\sum_{j=1}^{T} \sum_{(u, v) \in E} f((u, v), j)=\sum_{j=1}^{T} \sum_{(v, w) \in E} f((v, w), j)
$$

\footnotetext{
${ }^{2}$ We simplify the original definition to our context by setting all transmission times to 1 .
} 
- Flow value (source).

$$
\sum_{j=1}^{T}\left(\sum_{(s, v) \in E} f((s, v), j)-\sum_{(u, s) \in E} f((u, s), j)\right)=F
$$

- Flow value (sink).

$$
\sum_{j=1}^{T}\left(\sum_{(t, v) \in E} f((t, v), j)-\sum_{(u, t) \in E} f((u, t), j)\right)=-F
$$

In the usual flavor of dynamic flows, $T$ is given and the goal is to maximize $F$. In the quickest flow variant, the roles are reversed.

Definition 2.1 Given a flow network, the quickest flow for a given value $F$ is a dynamic flow $f$ satisfying (1-5) above with flow value $F$, such that the time horizon $T$ is minimal.

Theorem 2.1 ([9]) The quickest flow problem can be found in strongly polynomial time.

The Evacuation problem [21] is a variant of dynamic flow that we use, specifically the case of a single sink node [6]. In this problem, each node $v$ has an initial amount of $F(v)$ flow units, and the goal is to ship all the flow units to a single sink node $t$ in shortest possible time (every node $v$ with $F(v)>0$ is considered a source). Similarly to the single source case, shipment is described by a mapping $f: E \times[1, T] \rightarrow \mathbb{N}$ where $T$ is the time horizon. The dynamic flow is subject to the edge capacity constraints (Eq. 1), and the following additional constraints:

- Only initial and arriving flow can leave. For all $v \in V, j \in[1, T-1]$ :

$$
F(v)+\sum_{i=1}^{j} \sum_{(u, v) \in E} f((u, v), i) \geq \sum_{i=1}^{j+1} \sum_{(v, w) \in E} f((v, w), i)
$$

- Flow value (sources). For all $v \in V$ :

$$
\sum_{j=1}^{T}\left(\sum_{(v, w) \in E} f((v, w), j)-\sum_{(u, v) \in E} f((u, v), j)\right)=F(v)
$$

- Flow value (sink).

$$
\sum_{j=1}^{T}\left(\sum_{(u, t) \in E} f((u, t), j)-\sum_{(t, w) \in E} f((t, w), j)\right)=\sum_{v \in V} F(v)
$$

Formally, we use the following definition and result.

Definition 2.2 Given a flow network in which each node $v$ has value $F(v)$, a solution to the evacuation problem is a dynamic flow of multiple sources $f$ satisfying (1) and (6-8), such that the time horizon $T$ is minimal.

Theorem 2.2 ([6]) The evacuation problem can be found in strongly polynomial time. 


\subsection{Using Dynamic Flows in CWC}

In this section we show how to implement basic cloud access primitives using dynamic flow algorithms. These are the tasks of reading and writing to or from the cloud, invoked by a single node ( $\mathrm{cW}$ and cR), or by all nodes (cAW and cAR). Our goal in all the tasks and algorithms is to find a schedule that implements the task in the minimum amount of time.

Definition 2.3 Given a CWC model, a schedule for time interval I is a mapping that assigns, for each time step in I and each link $(u, v)$ : a send (or null) operation if $(u, v)$ is a local link, and a $F W$ or FR (or null) operation if $(u, v)$ is a cloud link.

We present optimal solutions to these problems in general directed graphs, using the quickest flow algorithm.

- Serving a Single Node: Let us consider cW first. We start with a lemma stating the close relation between schedules (Definition 2.3) implementing $\mathrm{cW}$ in our model and dynamic flows.

Lemma 2.3 Let $G=(V, E, w)$ be a graph in the $C W C$ model. Then there is a schedule implementing ${ } W_{i}$ from processing node $i$ to cloud node $v_{c}$ with message $S$ of size $s$ in $T$ rounds if and only if there is a dynamic flow of value $s$ and time horizon $T$ from source node $i$ to sink node $v_{c}$.

Proof: Converting a schedule to a dynamic flow is trivial, as send and receive operations between processing nodes and FW and FR operations directly translate to a dynamic flow that transports the same amount of flow while maintaining bandwidth capacities and resulting with a desired dynamic flow. For the other direction (converting a dynamic flow to a schedule), let $f$ be a dynamic flow of time horizon $T$ and value $s$ from node $i$ to the cloud $v_{c}$. We describe a schedule $A$ implementing $\mathrm{cW}_{i}$ as follows.

First, we construct another dynamic flow $f^{\prime}$ which is the same as $f$, except that no flow leaves the sink node $v_{c}$. Formally, let value $g(v, t)$ be the total number of flow units that are stored in node $v$ at time step $t \in\{0, T\}$ according to some dynamic flow $g$. Flow $f^{\prime}$ is constructed by induction; In time step $1, f^{\prime}$ is the same as $f$, except for setting $f^{\prime}(e, 1)=0$ for every edge $e$ that leaves $v_{c}$. Let $t \in\{1, T-1\}$. In step $t+1, f^{\prime}$ is defined the same as $f$, while truncating the sum of all flow that leaves node $v$ to be at most value $f^{\prime}(v, t)$ for every node $v$, and setting $f^{\prime}(e, 1)=0$ for every edge $e$ that leaves the sink.

Let $G_{p}=G-\left\{v_{c}\right\}$, and let value $_{g}\left(G_{p}, t\right)$ denote $\sum_{v \in V_{p}}$ value $_{g}(v, t)$ for some dynamic flow $g$. Initially, value $_{f}\left(G_{p}, 0\right)=$ value $_{f^{\prime}}\left(G_{p}, 0\right)=s$, and that by the induction, in every step $t \in\{1, T\}$, value $_{f}\left(G_{p}, t\right) \geq$ value $_{f^{\prime}}\left(G_{p}, t\right)$ due to flow units not being able to get to $G_{p}$ from $v_{c}$. Finally, in time step $T$, value va $_{f}\left(G_{p}, T\right)=0$ since all flow was sent to the sink, and thus value $f^{\prime}\left(G_{p}, T\right)=0$ as well and value $_{f^{\prime}}\left(v_{c}, T\right)=s$ due to flow conservation. Therefore, $f^{\prime}$ is a dynamic flow of time horizon (at most) $T$ and value $s$.

Now, we use $f^{\prime}$ to describe the volume of data that is sent along each link in every round of the schedule $A$, by translating flow between processing nodes to send/receive operations, and between processing and cloud nodes to FW operations (no FR operations are required according to $f^{\prime}$, because no flow leaves $v_{c}$ ). To specify which data is sent in every operation of the schedule, refer to all $\mathrm{FW}$ operations of the schedule. Let $K$ be the number of FW operations in $A$, let $i_{k}$ be the node initiating 
the $k$-th call to FW and let $l_{k}$ be the size of the message in that call. We assign the data transferred on each link during $A$ so that when node $i_{k}$ runs the $k$-th FW, it would write the part of $S$ starting at index $s \cdot \sum_{j=1}^{k-1} l_{j}$ and extending for $l_{k}$ bits. Note that processing nodes do not need to exchange indices of the data they transfer, as all nodes can calculate in preprocess time the schedule and thus "know in advance" the designated indices of the transferred data.

Correctness of the schedule $A$ follows from the validity of $f$, as well as its time complexity.

Theorem 2.4 Given any instance $G=(V, E, w)$ of the $C W C$ model, an optimal schedule realizing $c W_{i}$ can be computed in polynomial time.

Proof: Consider a $\mathrm{cW}$ issued by a processing node $i$, wishing to write $s$ bits to cloud node $v_{c}$. We construct an instance of quickest flow as follows. The flow network is $G$ where $w$ is the link capacity function, node $i$ is the source and $v_{c}$ is the sink. The requested flow value is $s$. The solution, computed by Theorem 2.1, is directly translatable to a schedule, after assigning index ranges to flow parts according to Lemma 2.3. Optimality of the resulting schedule follows from the optimality of the quickest flow algorithm.

\section{- Remarks.}

- Interestingly, in the presence of multiple cloud nodes, it may be the case that while writing to one cloud node, another cloud node is used as a relay station.

- Schedule computation can be carried out off-line: we can compute a schedule for each node $i$ and for each required file size $s$ (maybe it suffices to consider only powers of 2), so that in run-time, the initiating node would only need to tell all other nodes which schedule to use. ${ }^{3}$

Finally, we observe that the reduction sketched in the proof of Theorem 2.4 works for reading just as well: the only difference is reversing the roles of source and sink, i.e., pushing $s$ flow units from the cloud node $v_{c}$ to the requesting node $i$. We therefore have also:

Theorem 2.5 Given any instance of the $C W C$ model, an optimal schedule realizing $c R_{i}$ can be computed in polynomial time.

- Serving Multiple Nodes: Consider now operations with multiple invocations. Let us start with cAW (cAR is analogous, as mentioned above). Recall that in this task, each node has a (possibly empty) file to write to a cloud node. If all nodes write to the same cloud node, then using the evacuation problem variant of the quickest flow algorithm solves the problem (see Definition 2.2), However, if we need to write to multiple cloud nodes, we resort to the quickest multicommodity flow, defined as follows [34].

We are given a flow network as described in Sec. 2.1, but with $k$ source-sink pairs $\left\{\left(s_{i}, t_{i}\right)\right\}_{i=1}^{k}$, and $k$ demands $d_{1}, \ldots, d_{k}$. We seek $k$ flow functions $f_{i}$, where $f_{i}$ describes the flow of $d_{i}$ units of commodity $i$ from its source $s_{i}$ to its sink $t_{i}$, subject to the usual constraints: the edge capacity constraints $(1)$ applies to the sum of all $k$ flows, and the node capacity constraints (2-3), as well as the source and sink constraints (4-5) are written for each commodity separately.

For the case that all nodes write to the same cloud node, we can get the following theorem.

\footnotetext{
${ }^{3}$ We note that sending the initiating messages can be problematic if it uses cloud nodes as relays, and may require some sort of synchronization across all processing nodes.
} 
Theorem 2.6 Given any instance $G=(V, E, w)$ of the $C W C$ model, an optimal schedule realizing cAW (cAR) in which every node $i$ needs to write (read) a message of size $s_{i}$ to (from) cloud node $v_{c}$ can be computed in strongly polynomial time.

Proof: Similarly to Lemma 2.3, one can see that there is such an algorithm for cAW if and only if there is a dynamic flow solving the evacuation problem (Definition 2.2). Thus in order to solve cAW, we simply need to construct a flow network as is done in Theorem 2.4, and apply Theorem 2.2 in order to get the solution, that can be then translated to a schedule.

A schedule for cAR can be obtained by reversing the schedule for cAW, similarly to Theorem 2.5.

For the case of multiple targeted cloud nodes, it is known that determining whether there exists a feasible quickest multicommodity flow with a given time horizon $T$ is NP-hard, but on the positive side, there exists an FPTAS to it [15], i.e., we can approximate the optimal $T$ to within $1+\epsilon$, for any constant $\epsilon>0$. Extending the reduction for single commodity in the natural way, we obtain the following result.

Theorem 2.7 Given any instance of the CWC model and $\epsilon>0$, a schedule realizing cAW or cAR can be computed in time polynomial in the instance size and $\epsilon^{-1}$. The length of the schedule is at most $(1+\epsilon)$ times larger than the optimal length.

\section{Computing \& Writing Combined Values}

Flow-based techniques are not applicable in the case of writing a combined value, because the very essence of combining violates conservation constraints (i.e., the number of bits entering a node may be different than the number of bits leaving it). However, in Sec. 3.1 we explain how to implement cComb in the general case using cAW and cAR. While simple and generic, these implementations can have time complexity much larger than optimal. We offer partial remedy in Sec. 3.2, where we present our main result: an algorithm for cComb when $\otimes$ is commutative and the local network has "fat links," i.e., all local links have capacity at least $s$. For this important case, we show how to complete the task in time larger than the optimum by an $O\left(\log ^{2} n\right)$ factor.

\subsection{Combining in General Graphs}

We now present algorithms for cComb and for cCast on general graphs, using the primitives treated in Sec. 2. Note that with a non-commutative operator, the operands must be ordered; using renaming if necessary, we assume w.l.o.g. that in such cases the nodes are indexed by the same order of their operands.

Theorem 3.1 Let $T_{s}$ be the running time of cAW (and cAR) when all files have size s. Then Alg. 1 solves cComb in $O\left(T_{s} \log n\right)$ rounds.

Proof: By algorithm. The idea is to do the combining over a binary "computation tree" by using the cloud to store the partial results. The computation tree is defined as follows (see Fig. 2). Let 

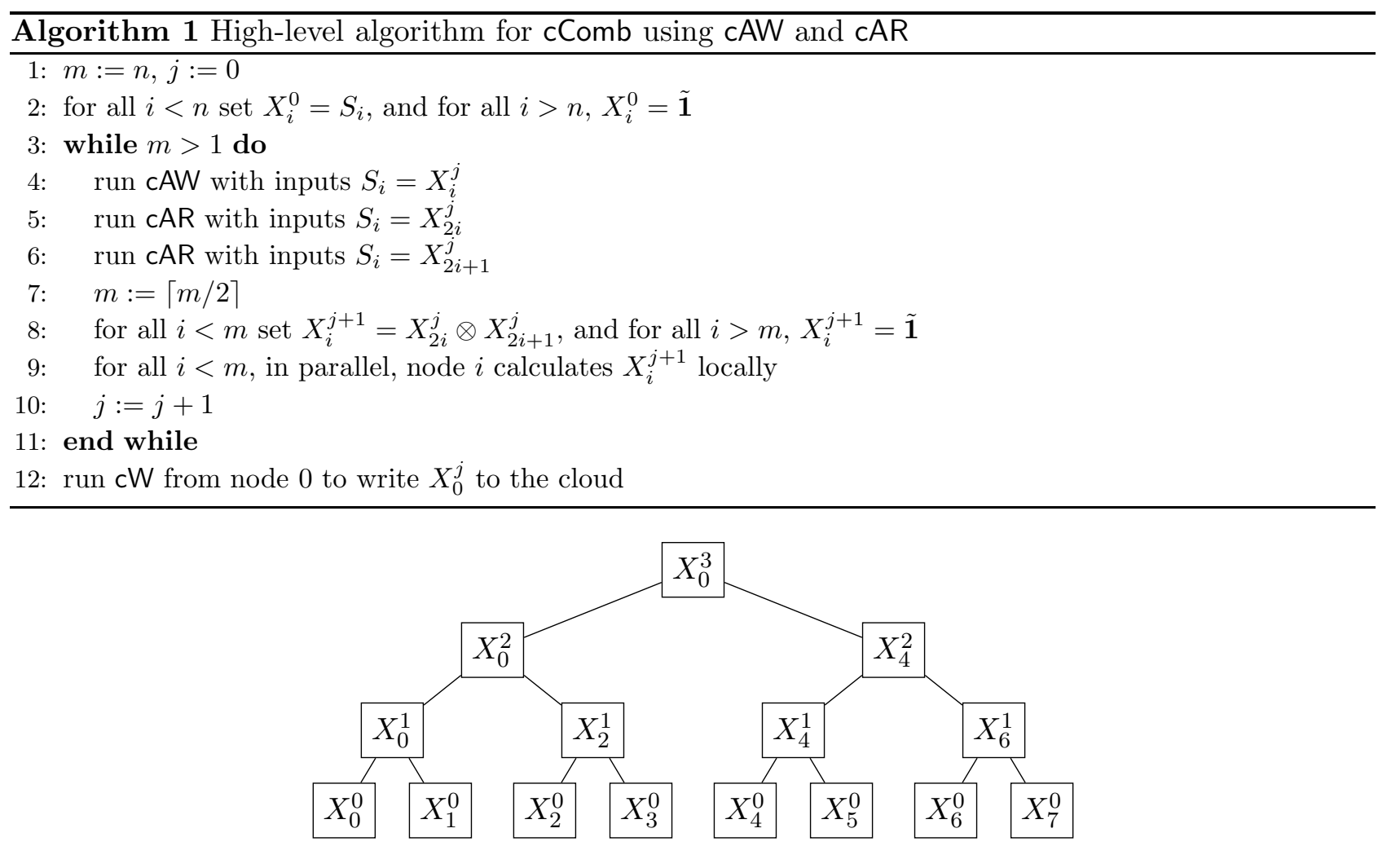

Figure 2: Computation tree with $n=8 . X_{i}^{j}$ denotes the result stored in node $i$ in iteration $j$.

$X_{i}^{j}$ denote the $i$-th node at level $j$, as well as the value of that node. The leaves $X_{i}^{0}$ are the input values, and the value of an internal node $X_{i}^{j+1}$ at level $j+1$ with left child $X_{2 i}^{j}$ and right child $X_{2 i+1}^{j}$ is $X_{2 i}^{j} \otimes X_{2 i+1}^{j}$. Pseudocode is provided in Alg. 1. Correctness of the algorithm follows from the observation that after each execution of Step 4, there are $m$ files of size $s$ written in the cloud, whose product is the required output, and that $m$ is halved in every iteration. If at any iteration $m$ is odd, then node $\lceil m / 2\rceil-1$ only needs to read one file, and therefore we set the other file that it reads to be $\tilde{\mathbf{1}}$. When $m$ reaches 1 , there is only 1 file left, which is the required result.

As for the time analysis: Clearly, a single iteration of the while loop takes $3 T_{s}=O\left(T_{s}\right)$ rounds. Note that when writing $\tilde{\mathbf{1}}$ to the cloud, it can be encoded as a 0-bit string, which can only improve the runtime. There are $\lceil\log n\rceil$ rounds due to Step 7, and Step 12 is also completed in $O\left(T_{s}\right)$ rounds, and thus the total runtime of the algorithm is $O\left(T_{s} \log n\right)$.

In a way, cCast is the "reverse" problem of cComb, since it starts with $s$ bits in the cloud and ends with $s$ bits of output in every node. However, cCast is easier than cComb because our model allows for concurrent reads and disallows concurrent writes. We have the following result.

Theorem 3.2 Let $T_{s}$ be the time required to solve cAR when all files have size s. Then cCast can be solved in $T_{s}$ rounds as well.

Proof: First note that if there were $n$ copies of the input file $S$ in the cloud, then cCast and cAR would have been the exact same problem. The theorem follows from the observation that any algorithm for 


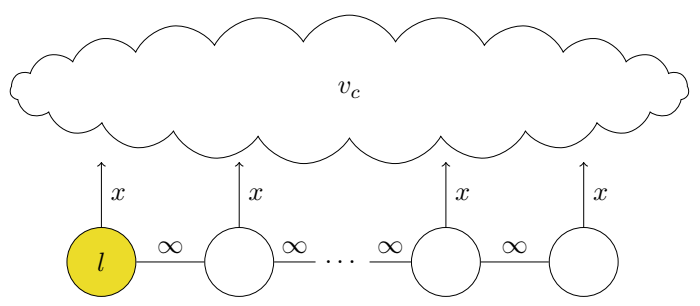

Figure 3: A simple path example. The optimal distance to travel in order to write an s-bits file to the cloud would be $\sqrt{s / x}$.

cAR with $n$ inputs of size $s$ in the cloud can be modified so that each invocation of FR with argument $S_{i}$ is converted to FR with argument $S$ (the input of cCast).

\subsection{Combining Commutative Operators in Fat links Network}

In the case of $s$-fat-links network (i.e., all local links are have bandwidth at least $s$, and all links are symmetric) we can construct a near-optimal algorithm for cComb. The idea is to use multiple $\mathrm{cW}$ and $\mathrm{cR}$ operations instead of cAW and cAR. The challenge is to minimize the number of concurrent operations per node; to this end we use sparse covers [5].

We note that if the network is $s$-fat-links but the operand size is $s^{\prime}>s$, the algorithms still apply, with an additional factor of $\left\lceil s^{\prime} / s\right\rceil$ to the running time. The lower bounds in this section, however, may change by more than that factor.

We start with a tight analysis of $\mathrm{cW}$ and $\mathrm{cR}$ in this setting and then generalize to cComb and cCast.

Implementation of $\mathbf{c W}$ and $\mathbf{c R}$. Consider $\mathrm{cW}_{i}$, where $i$ wishes to write $s$ bits to a given cloud node. The basic tension in finding an optimal schedule for $\mathrm{cW}_{i}$ is that in order to use more cloud bandwidth, more nodes need to be enlisted. But while more bandwidth reduces the transmission time, reaching remote nodes (that provide the extra bandwidth) increases the traversal time. Our algorithm looks for the sweet spot where the conflicting effects are more-or-less balanced.

For example, consider a simple path of $n$ nodes with infinite local bandwidth, where each node is connected to the cloud with bandwidth $x$ (Fig. 3). Suppose that the leftmost node $l$ needs to write a message of $s$ bits to the cloud. By itself, writing requires $s / x$ rounds. Using all $n$ nodes, uploading would take $O(s / n x)$ rounds, but $n-1$ rounds are needed to ship the messages to the fellow-nodes. The optimal solution in this case is to use only $\sqrt{s / x}$ nodes: the time to ship the file to all these nodes is $\sqrt{s / x}$, and the upload time is $\frac{s / \sqrt{s / x}}{x}=\sqrt{s / x}$, because each node needs to upload only $s / \sqrt{s / x}$ bits.

In general, we define "cloud clusters" to be node sets that optimize the ratio between their diameter and their total bandwidth to the cloud. Our algorithms for cW and cR use nodes of cloud clusters. We prove that the running-time of our implementation is asymptotically optimal. Formally, we have the following. 
Definition 3.1 Let $G=(V, E, w)$ be a $C W C$ system with processor nodes $V_{p}$ and cloud nodes $V_{c}$. The cloud bandwidth of a processing node $i \in V_{p}$ w.r.t. a given cloud node $v_{c} \in V_{c}$ is $b_{c}(i) \stackrel{\text { def }}{=} w\left(i, v_{c}\right)$. A cluster $B \subseteq V_{p}$ in $G$ is a connected set of processing nodes. The cloud (up or down) bandwidth of cluster $B$ w.r.t a given cloud node, denoted $b_{c}(B)$, is the sum of the cloud bandwidth to $v_{c}$ over all nodes in $B: b_{c}(B) \stackrel{\text { def }}{=} \sum_{i \in B} b_{c}(i)$. The (strong) diameter of cluster $B$, denoted $\operatorname{diam}(B)$, is the maximum distance between any two nodes of $B$ in the induced graph $G[B]: \operatorname{diam}(B)=\max _{u, v \in B} \operatorname{dist}_{G[B]}(u, v)$.

We use the following definition for the network when ignoring the cloud. Note that the metric here is hop-based- $w$ indicates link bandwidths.

Definition 3.2 Let $G=(V, E, w)$ be a $C W C$ system with processing nodes $V_{p}$ and cloud nodes $V_{c}$. The ball of radius $r$ around node $i \in V_{p}$, denoted $B_{r}(i)$ is the set of nodes at most $r$ hops away from $i$ in $G_{p}$.

Finally, we define the concept of cloud cluster of a node.

Definition 3.3 Let $G=(V, E, w)$ be a $C W C$ system with processing nodes $V_{p}$ and cloud node $v_{c}$, and let $i \in V_{p}$. Given $s \in \mathbb{N}$, the $s$-cloud radius of node $i$, denoted $k_{s}(i)$, is defined to be

$$
k_{s}(i) \stackrel{\text { def }}{=} \min \left(\left\{\operatorname{diam}\left(G_{p}\right)\right\} \cup\left\{k \mid(k+1) \cdot b_{c}\left(B_{k}(i)\right) \geq s\right\}\right) .
$$

The ball $B_{i} \stackrel{\text { def }}{=} B_{k_{s}(i)}(i)$ is the s-cloud cluster of node $i$. The timespan of the s-cloud cluster of $i$ is denoted $Z_{i} \stackrel{\text { def }}{=} k_{s}(i)+\frac{s}{b_{c}\left(B_{i}\right)}$. We sometimes omit the s qualifier when it is clear from the context.

In words, $B_{i}$ is a cluster of radius $k(i)$ around node $i$, where $k(i)$ is the smallest radius that allows writing $s$ bits to $v_{c}$ by using all cloud bandwidth emanating from $B_{i}$ for $k(i)+1$ rounds. $Z_{i}$ is the time required (1) to send $s$ bits from node $i$ to all nodes in $B_{i}$, and (2) to upload $s$ bits to $v_{c}$ collectively by all nodes of $B_{i}$. Note that $B_{i}$ is easy to compute. We can now state our upper bound.

Theorem 3.3 Given a fat-links $C W C$ system, Alg. 2 solves the s-bits $\mathrm{cW}_{i}$ problem in $O\left(Z_{i}\right)$ rounds on $B_{i}$.

Proof: The algorithm broadcasts all $s$ bits to all nodes in $B_{i}$, and then each node writes a subrange of the data whose size is proportional to its cloud bandwidth. Correctness is obvious. As for the time analysis: Steps 1-2 require $O(k(i))$ rounds. In the loop of steps $4-5, b_{c}\left(B_{i}\right)$ bits are sent in every round, and thus it terminates in $O\left(s / b_{c}\left(B_{i}\right)\right)$ rounds. The theorem follows from the definition of $Z_{i}$.

Next, we show that our solution for $\mathrm{cW}_{i}$ is optimal, up to a constant factor. We consider the case of an incompressible input string: such a string exists for any size $s \in \mathbb{N}$ (see, e.g., [27]). As a consequence, in any execution of a correct algorithm, $s$ bits must cross any cut that separates $i$ from the cloud node, giving rise to the following lower bound.

Theorem 3.4 Any algorithm solving $\mathrm{cW}_{i}$ in a fat-links $C W C$ requires $\Omega\left(Z_{i}\right)$ rounds.

Proof: By definition, $Z_{i}=k(i)+s / b_{c}\left(B_{i}\right)$. Lemma 3.5 and Lemma 3.6 show that each term of $Z_{i}$ is a lower bound on the running time of any algorithm for $\mathrm{cW}_{i}$.

Lemma 3.5 Any algorithm solving $\mathrm{cW}_{i}$ in a fat-links $C W C$ system requires $\Omega(k(i))$ rounds. 


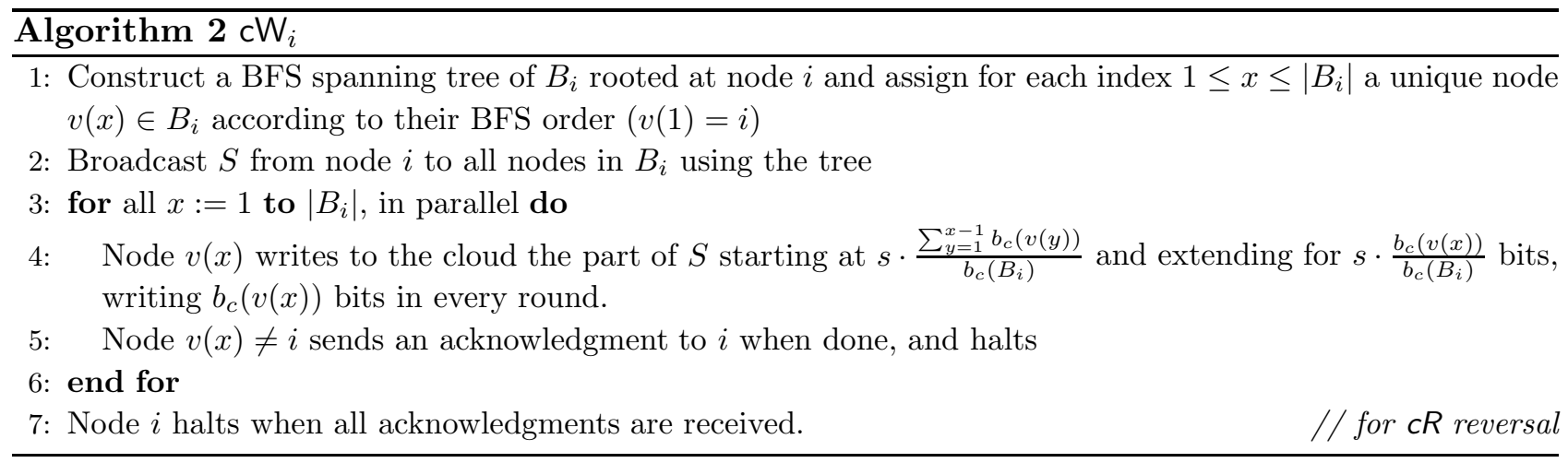

Proof: Let $A$ be an algorithm for $\mathrm{cW}_{i}$ that writes string $S$ in $t_{A}$ rounds. If $t_{A} \geq \operatorname{diam}\left(G_{p}\right)$ then $t_{A} \geq k(i)$ and we are done. Otherwise, we count the number of bits of $S$ that can get to the cloud in $t_{A}$ rounds. Since $S$ is initially stored in $i$, in a given round $t$, only nodes in $B_{t-1}(i)$ can write pieces of $S$ to the cloud. Therefore, overall, $A$ writes to the cloud at most $\sum_{t=1}^{t_{A}} b_{c}\left(B_{t-1}(i)\right) \leq t_{A} \cdot b_{c}\left(B_{t_{A}-1}(i)\right)$ bits. Hence, by assumption that $A$ solves $\mathrm{cW}_{i}$, we must have $t_{A} \cdot b_{c}\left(B_{t_{A}-1}(i)\right) \geq s$. The lemma now follows from definition of $k(i)$ as the minimal integer $\ell \leq \operatorname{diam}\left(G_{p}\right)$ such that $(\ell+1) \cdot b_{c}\left(B_{\ell}(i)\right) \geq s$ if it exists (otherwise, $k_{c}(i)=\operatorname{diam}\left(G_{p}\right)$ and $t_{A}>\operatorname{diam}\left(G_{p}\right)$ ). Either way, we are done.

Lemma 3.6 Any algorithm solving $\mathrm{cW}_{i}$ in a fat-links $C W C$ system requires $\Omega\left(s / b_{c}\left(B_{i}\right)\right)$ rounds.

Proof: Let $A$ be an algorithm that solves $\mathrm{cW}_{i}$ in $t_{A}$ rounds. If $k(i)=\operatorname{diam}\left(G_{p}\right)$ then $B_{i}$ contains all processing nodes $V_{p}$, and the claim is obvious, as no more than $b_{c}\left(V_{p}\right)$ bits can be written to the cloud in a single round. Otherwise, $k(i) \geq s / b_{c}\left(B_{i}\right)-1$ by Definition 3.3, and we are done since $t_{A}=\Omega(k(i))$ by Lemma 3.5 .

By reversing time (and hence information flow) in a schedule of $\mathrm{cW}$, one gets a schedule for $\mathrm{cR}$. Hence we have the following immediate corollaries.

Theorem $3.7 c R_{i}$ can be executed in $O\left(Z_{i}\right)$ rounds in a fat-links $C W C$.

Theorem $3.8 c R_{i}$ in a fat-links $C W C$ requires $\Omega\left(Z_{i}\right)$ rounds.

- Remark: The lower bound of Theorem 3.4 and the definition of cloud clusters (Definition 3.3) show an interplay between the message size $s$, cloud bandwidth, and the network diameter; For large enough $s$, the cloud cluster of a node includes all processing nodes (because the time spent crossing the local network is negligible relative to the upload time), and for small enough $s$, the cloud cluster includes only the invoking node, rendering the local network redundant.

Implementation of cComb. Below, we first show how to implement cComb using any given cover. In fact, we shall use sparse covers [5], which allow us to to get near-optimal performance.

Intuitively, every node $i$ has a cloud cluster $B_{i}$ which allows it to perform $\mathrm{cW}_{i}$, and calculating the combined value within every cloud cluster $B_{i}$ is straight-forward (cf. Alg. 4 and Lemma 3.11). Therefore, given a partition of the graph that consists of pairwise disjoint cloud clusters, cComb can be solved by combining the inputs in every cloud cluster, followed by combining the partial results in a computation-tree fashion using $\mathrm{cW}$ and $\mathrm{cR}$. However, such a partition may not always exist, and we 


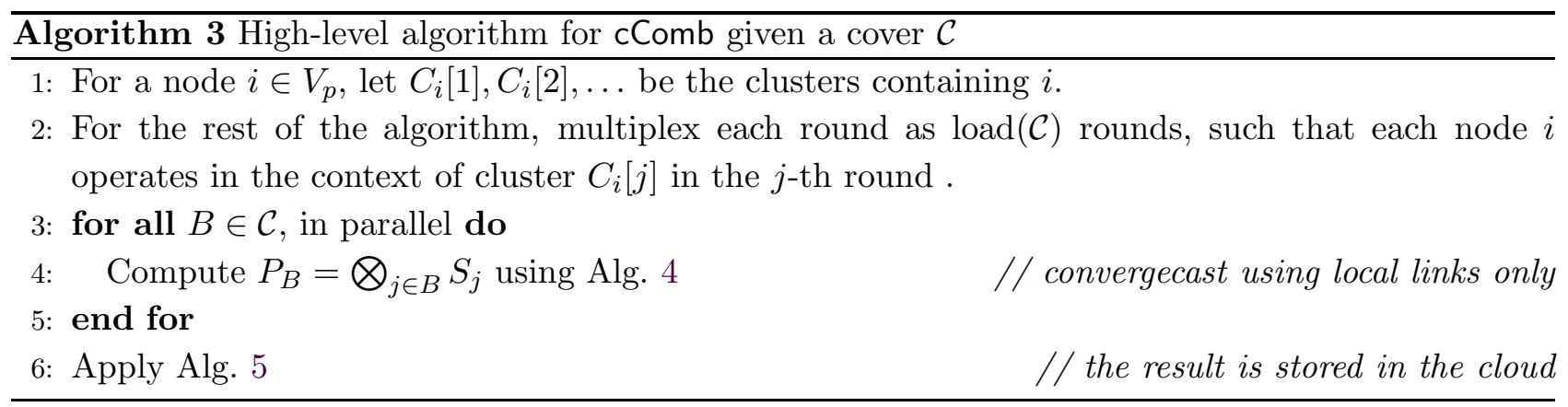

resort to a cover of the nodes. Given a cover $\mathcal{C}$ in which every node is a member of at $\operatorname{most} \operatorname{load}(\mathcal{C})$ clusters, we can use the same technique, while increasing the running-time by a factor of $\operatorname{load}(\mathcal{C})$ by time multiplexing. Using Awerbuch and Peleg's sparse covers (see Theorem 3.16), we can use an initial cover $\mathcal{C}$ that consists of all cloud clusters in the graph to construct another cover, $\mathcal{C}^{\prime}$, in which $\operatorname{load}\left(\mathcal{C}^{\prime}\right)$ is $O(\log n)$, paying an $O(\log n)$ factor in cluster diameters, and use $\mathcal{C}^{\prime}$ to get near-optimal results.

Definition 3.4 Let $G$ be a $C W C$ system, and let $B$ be a cluster in $G$ (see Definition 3.1). The timespan of node $i$ in $B$, denoted $Z_{B}(i)$, is the minimum number of rounds required to perform $c W_{i}$ (or $\left.c R_{i}\right)$, using only nodes in $B$. The timespan of cluster $B$, denoted $Z(B)$, is given by $Z(B)=\min _{i \in B} Z_{B}(i)$. The leader of cluster $B$, denoted $r(B)$, is a node with minimal timespan in $B$, i.e., $r(B)=\operatorname{argmin}_{i \in B} Z_{B}(i)$.

In words, the timespan of cluster $B$ is the minimum time required for any node in $B$ to write an $s$-bit string to the cloud using only nodes of $B$.

From Theorem 3.3, we get the following lemma:

Lemma 3.9 For every node $i, Z\left(B_{i}\right) \leq Z_{B_{i}}(i)=O\left(Z_{i}\right)$.

Definition 3.5 Let $G$ be a $C W C$ system with processing node set $V_{p}$. A cover of $G$ is a set of clusters $\mathcal{C}=\left\{B_{1}, \ldots, B_{m}\right\}$ such that $\cup_{B \in \mathcal{C}} B=V_{p}$. The load of node $i$ in a cover $\mathcal{C}$ is the number of clusters in $\mathcal{C}$ that contain $i$, i.e., $\operatorname{load}_{\mathcal{C}}(i)=|\{B \in \mathcal{C} \mid i \in B\}|$. The load of cover $\mathcal{C}$ is the maximum load of any node in the cover, i.e.. $\operatorname{load}(\mathcal{C})=\max _{i \in V_{p}} \operatorname{load}_{\mathcal{C}}(i)$. The timespan of cover $\mathcal{C}$, denoted $Z(\mathcal{C})$, is the maximum timespan of any cluster in $\mathcal{C}, Z(\mathcal{C})=\max _{B \in \mathcal{C}} Z(B)$. The diameter of cover $\mathcal{C}$, denoted $\operatorname{diam}_{\max }(\mathcal{C})$, is the maximum diameter of any cluster in $\mathcal{C}, \operatorname{diam}_{\max }(\mathcal{C})=\max _{B \in \mathcal{C}} \operatorname{diam}(B)$.

We now give an upper bound in terms of any given cover $\mathcal{C}$.

Theorem 3.10 Alg. 3 solves cComb in a fat-links $C W C$ in $O\left(\operatorname{diam}_{\max }(\mathcal{C}) \cdot \operatorname{load}(\mathcal{C})+Z(\mathcal{C}) \cdot \operatorname{load}(\mathcal{C}) \cdot \log |\mathcal{C}|\right)$ rounds.

The basic strategy is to first compute the combined value in each cluster using only the local links, and then combine the cluster values using a computation tree. However, unlike Alg. 1, we use cW and cR instead of cAW and cAR.

A high-level description is given in Alg. 3. The algorithm consists of a preprocessing part (lines 1-2), and the execution part, which consists of the "low-level" computation using only local links (lines 3-5), and the "high-level" computation among clusters (line 6). We elaborate on each below. 


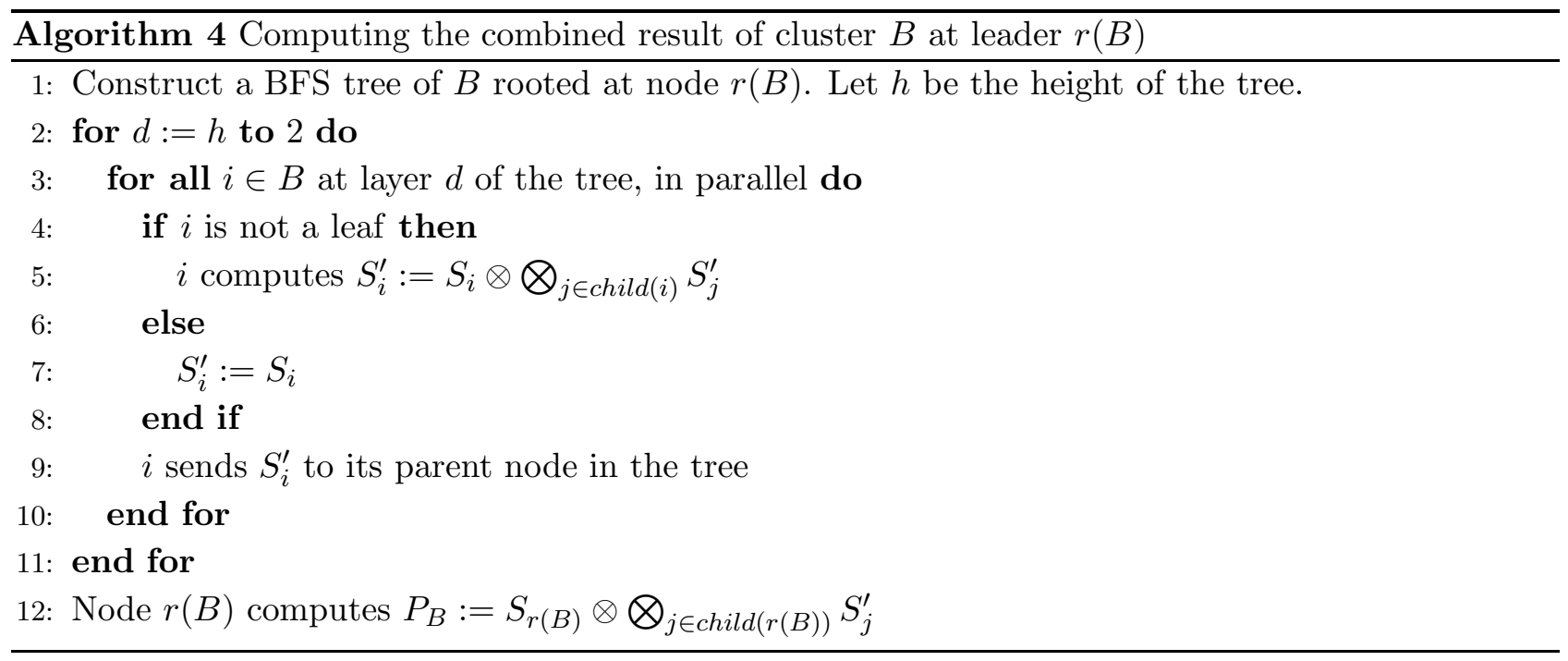

- Preprocessing. A major component of the preprocessing stage is computing the cover $\mathcal{C}$, which we specify later (see Theorem 3.15). In Alg. 3 we describe the algorithm as if it operates in each cluster independently of other clusters, but clusters may overlap. To facilitate this mode of operation, we use time multiplexing: nodes execute work on behalf of the clusters they are member of in a round-robin fashion, as specified in lines 1-2 of Alg. 3. This allows us to invoke operations limited to clusters in all clusters "simultaneously" by increasing the time complexity by a $\operatorname{load}(\mathcal{C})$ factor.

- Low levels: Combining within a single cluster. To implement line 4 of Alg. 3, we build, in each cluster $B \in \mathcal{C}$, a spanning tree rooted at $r(B)$, and apply Convergecast [33] using $\otimes$. Ignoring the multiplexing of Alg. 3, we have:

Lemma 3.11 Alg. 4 computes $P_{B}=\bigotimes_{i \in B} S_{i}$ at node $r(B)$ in $O(\operatorname{diam}(B))$ rounds.

To get the right overall result, each input $S_{i}$ is associated with a single cluster in $\mathcal{C}$. To this end, we require each node to select a single cluster in which it is a member as its home cluster. When applying Alg. 4, we use the rule that the input of node $i$ in a cluster $B \ni i$ is $S_{i}$ if $B$ is $i$ 's home cluster, and $\tilde{\mathbf{1}}$ otherwise.

Considering the scheduling obtained by Step 2, we get the following lemma.

Lemma 3.12 Steps 3-5 of Alg. 3 terminate in $O\left(\operatorname{diam}_{\max }(\mathcal{C}) \cdot \operatorname{load}(\mathcal{C})\right)$ rounds, with $P_{B}$ stored at the leader node of $B$ for each cluster $B \in C$.

- High levels: Combining using the cloud. When Alg. 3 reaches Step 6, the combined result of every cluster is stored in the leader of the cluster. The idea is now to fill in a computation tree whose leaves are these values (see Fig. 2).

We combine the partial results by filling in the values of a computation tree defined over the clusters. The leaves of the tree are the combined values of the clusters of $\mathcal{C}$, as computed by Alg. 4 . To fill in the values of other nodes in the computation tree, we use the clusters of $\mathcal{C}$ : Each node in the tree is assigned a cluster which computes its value using the $\mathrm{cR}$ and $\mathrm{cW}$ primitives.

Specifically, in Alg. 5 we consider a binary tree with $|\mathcal{C}|$ leaves, where each non-leaf node has 


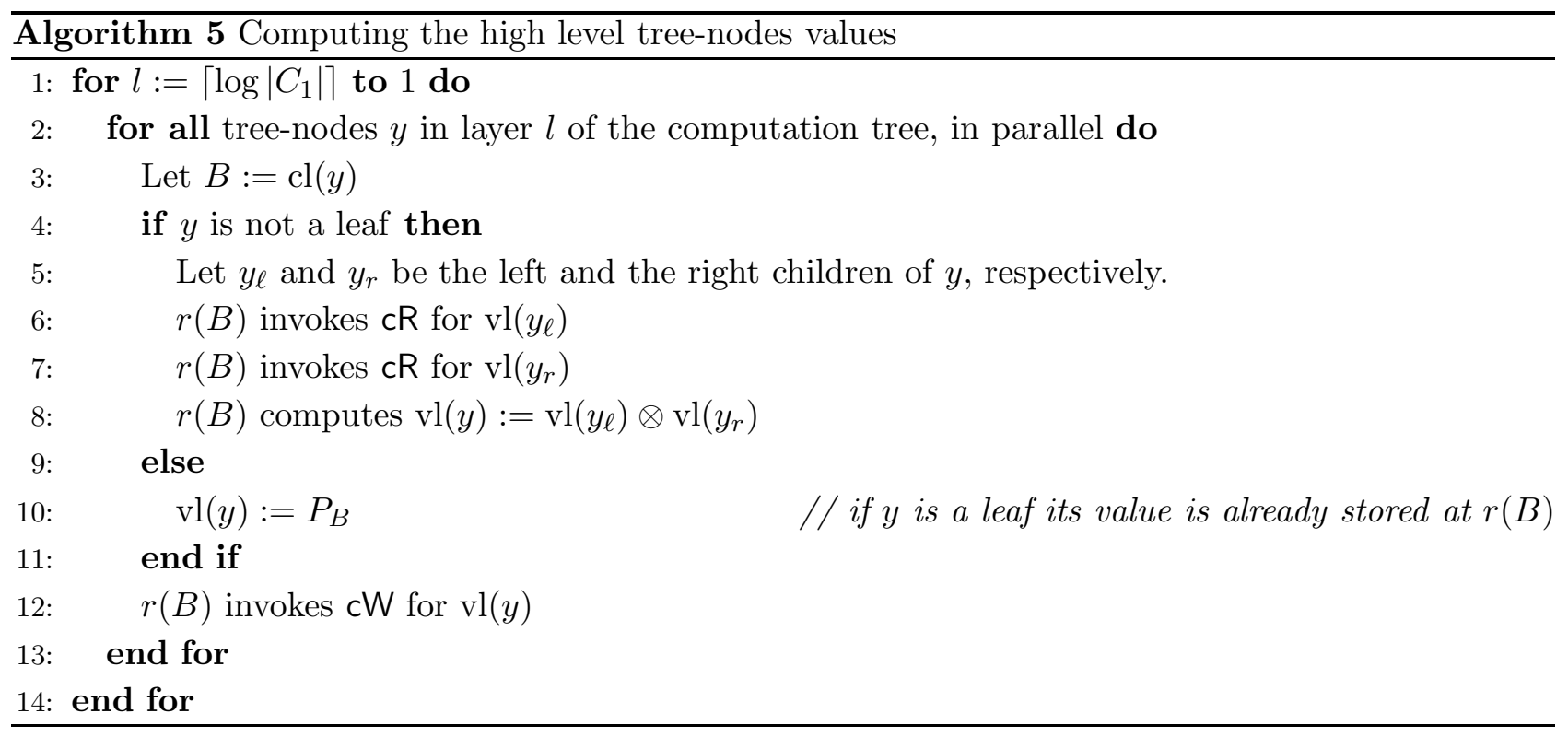

exactly two children. The tree is constructed from a complete binary tree with $2^{\lceil\log |\mathcal{C}|\rceil}$ leaves, after deleting the rightmost $2^{\lceil\log |\mathcal{C}|\rceil}-|\mathcal{C}|$ leaves. (If by the end the rightmost leaf is the only child of its parent, we delete the rightmost leaf repeatedly until this is not the case.)

We associate each node $y$ in the computation tree with a cluster $\operatorname{cl}(y) \in \mathcal{C}$ and a value $\operatorname{vl}(y)$, computed by the processors in $\operatorname{cl}(y)$ are responsible to compute $\operatorname{vl}(y)$. Clusters are assigned to leaves by index: The $i$-th leaf from the left is associated with the $i$-th cluster of $\mathcal{C}$. For internal nodes, we assign the clusters arbitrarily except that we ensure that no cluster is assigned to more than one internal node. (This is possible because in a tree where every node has two or no children, the number of internal nodes is smaller than the number of leaves.)

The clusters assigned to tree nodes compute the values as follows (see Alg. 5). The value associated with a leaf $y_{B}$ corresponding to cluster $B$ is $\operatorname{vl}\left(y_{B}\right)=P_{B}$. This way, every leaf $x$ has $\operatorname{vl}(x)$, stored in the leader of $\operatorname{cl}(x)$, which can write it to the cloud using $\mathrm{cW}$. For an internal node $y$ with children $y_{l}$ and $y_{r}$, the leader of $\operatorname{cl}(y)$ obtains $\operatorname{vl}\left(y_{l}\right)$ and $\operatorname{vl}\left(y_{r}\right)$ using $\mathrm{cR}$, computes their product $\operatorname{vl}(y)=\operatorname{vl}\left(y_{l}\right) \otimes \operatorname{vl}\left(y_{r}\right)$ and invokes $\mathrm{cW}$ to write it to the cloud. The executions of $\mathrm{cW}$ and $\mathrm{cR}$ in a cluster $B$ are done by the processing nodes of $B$.

Computation tree values are filled layer by layer, bottom up. With this strategy, we have the following result.

Lemma 3.13 Alg. 5 computes $\bigotimes_{i=1}^{m} P_{i}$ in $O(Z(\mathcal{C}) \cdot \log |\mathcal{C}|)$ rounds, assuming that all clusters operate in complete parallelism.

Proof: Computing all values in a tree layer requires a constant number of $\mathrm{cW}$ and $\mathrm{cR}$ invocations in a cluster, i.e., by Definition 3.5, at most $O(Z(\mathcal{C}))$ rounds of work in every layer. The number of layers is $\lceil\log |\mathcal{C}|\rceil$. the result follows.

From Lemma 3.13, considering the multiplexing of Step 2, we get the following result.

Lemma 3.14 Line 6 of Alg. 3 is completed in $O(Z(\mathcal{C}) \cdot \operatorname{load}(\mathcal{C}) \cdot \log |\mathcal{C}|)$ rounds. 
We can therefore conclude:

Proof of Theorem 3.10: By Lemma 3.12 and Lemma 3.14.

- Remark. We note that in Alg. 5, Lines 6, 7 and 12 essentially compute cAR and cAW in which only the relevant cluster leaders have inputs. Therefore, these calls can be replaced with a collective call for appropriate cAR and cAW, making the multiplexing of Line 2 of Alg. 3 unnecessary (similarly to Alg. 1). By using optimal schedules for cAW and cAR, the running-time can only improve beyond the upper bound of Theorem 3.10.

Sparse Covers. We now arrive at our main result, derived from Theorem 3.10 using a particular flavor of covers. The result is stated in terms of the maximal timespan of a graph, according to the following definition.

Definition 3.6 Let $G=(V, E, w)$ be a $C W C$ system with fat links. $Z_{\max } \stackrel{\text { def }}{=} \max _{i \in V_{p}} Z_{i}$ is the maximal timespan in $G$.

In words, $Z_{\max }$ is the maximal amount of rounds that is required for any node in $G$ to write an $s$-bit message to the cloud, up to a constant factor (cf. Theorem 3.4).

Theorem 3.15 Let $G=(V, E, w)$ be a $C W C$ system with fat links. Then cComb with a commutative combining operator can be solved in $O\left(Z_{\max } \log ^{2} n\right)$ rounds.

To prove Theorem 3.15 we use sparse covers. We state the result from [5].

Theorem 3.16 ([5]) Given any cover $\mathcal{C}$ and an integer $\kappa \geq 1$, a cover $\mathcal{C}^{\prime}$ that satisfies the following properties can be constructed in polynomial time.

(i) For every cluster $B \in \mathcal{C}$ there exists a cluster $B^{\prime} \in \mathcal{C}^{\prime}$ such that $B \subseteq B^{\prime}$.

(ii) $\max _{B^{\prime} \in \mathcal{C}^{\prime}} \operatorname{diam}\left(B^{\prime}\right) \leq 4 \kappa \max _{B \in \mathcal{C}} \operatorname{diam}(B)$

(iii) $\operatorname{load}\left(\mathcal{C}^{\prime}\right) \leq 2 \kappa|\mathcal{C}|^{1 / \kappa}$.

Proof of Theorem 3.15: Let $\mathcal{C}$ be the cover defined as the set of all cloud clusters in the system. By applying Theorem 3.16 to $\mathcal{C}$ with $\kappa=\lceil\log n\rceil$, we obtain a cover $\mathcal{C}^{\prime}$ with $\operatorname{load}\left(\mathcal{C}^{\prime}\right) \leq 4\lceil\log n\rceil$ because $|\mathcal{C}| \leq n$. By ii, $\operatorname{diam}_{\max }\left(\mathcal{C}^{\prime}\right) \leq 4\lceil\log n\rceil \cdot \operatorname{diam}_{\max }(\mathcal{C})$. Now, let $B^{\prime} \in \mathcal{C}^{\prime}$. We can assume w.l.o.g. that there is a cluster $B \in \mathcal{C}$ such that $B \subseteq B^{\prime}$ (otherwise $B^{\prime}$ can be removed from $\mathcal{C}^{\prime}$ ). $B$ is a cloud cluster of some node $i \in B^{\prime}$, and therefore by Lemma 3.9 and by Definition 3.4, we get that $Z\left(B^{\prime}\right) \leq Z(B)=O\left(Z_{i}\right)=O\left(Z_{\max }\right)$. Since this bound holds for all clusters of $\mathcal{C}^{\prime}, Z\left(\mathcal{C}^{\prime}\right)=O\left(Z_{\max }\right)$.

An $O\left(\operatorname{diam}_{\max }(\mathcal{C}) \cdot \log ^{2} n+Z_{\max } \cdot \log ^{2} n\right)$ time bound for cComb is derived by applying Theorem 3.10 to cover $\mathcal{C}^{\prime}$. Finally, let $B_{j} \in \mathcal{C}$ be a cloud cluster of diameter $\operatorname{diam}_{\text {max }}(\mathcal{C})$. Recall that by Definition 3.3, $\operatorname{diam}\left(B_{j}\right) \leq 2 k(j) \leq 2 Z_{j} \leq 2 Z_{\max }$. We therefore obtain an upper bound of $O\left(Z_{\max } \log ^{2} n\right)$ rounds.

We close with a lower bound.

Theorem 3.17 Let $G=(V, E, w)$ be a $C W C$ system with fat links. Then cComb requires $\Omega\left(Z_{\max }\right)$ rounds.

Proof: By reduction from $\mathrm{cW}$. Let $i$ be any processing node. Given $S$, assign $S_{i}=S$ as the input of 
node $i$ in cComb, and for every other node $j \neq i$, assign $S_{j}=\tilde{\mathbf{1}}$. Clearly, any algorithm for cComb that runs with these inputs solves $\mathrm{cW}_{i}$ with input $S$. The result follows from Theorem 3.4.

cCast. To implement cCast, one can reverse the schedule of cComb. However, a slightly better implementation is possible, because there is no need to ever write to the cloud node. More specifically, let $\mathcal{C}$ be a cover of $V_{p}$. In the algorithm for cCast, each cluster leader invokes $\mathrm{cR}$, and then the leader disseminates the result to all cluster members. The time complexity for a single cluster $B$ is $O(Z(B))$ for the cR operation, and $O(\operatorname{diam}(B))$ rounds for the dissemination of $S$ throughout $B$ (similarly to Lemma 3.11). Using the multiplexing to $\operatorname{load}(\mathcal{C})$ as in in Step 2 of Alg. 3, we obtain the following result.

Theorem 3.18 Let $G=(V, E, w)$ be a $C W C$ system with fat links. Then cCast can be performed in $O\left(Z_{\max } \cdot \log ^{2} n\right)$ rounds.

Finally, we note that since any algorithm for cCast also solves $\mathrm{cR}_{i}$ problem for every node $i$, we get from Theorem 3.8 the following result.

Theorem 3.19 Let $G=(V, E, w)$ be a CWC system with fat links. Any algorithm solving cCast requires $\Omega\left(Z_{\max }\right)$ rounds.

\section{Non-Commutative Operators and the Wheel Settings}

In this section we consider cComb for non-commutative operators in the wheel topology (Fig. 1).

Trivially, Alg. 3 and Theorem 3.10 apply in the non-commutative case if the ordering of the nodes happens to match an ordering induced by the algorithm, but this need not be the case in general. However, it seems reasonable to assume that processing nodes are physically connected according to their combining order. Neglecting other possible connections, assuming that the last node is also connected to the first node for symmetry, and connecting a cloud node to all processors, we arrive at the wheel topology, which we study in this section.

Our main result in this section is an algorithm for cComb for arbitrary wheel topology that works in time which is a logarithmic factor larger than optimal. In contrast to the result of Theorem 3.15 that applies only to graphs with fat links, here we analyze the wheel topology with arbitrary bandwidths (assuming symmetric links). We note that by using standard methods [24], the algorithm presented in this section can be extended to compute, with the same asymptotic time complexity, all prefix sums, i.e., compute, for each $0 \leq j<n, \bigotimes_{i=0}^{j} S_{i}$.

We distinguish between holistic and modular combining operators, defined as follows. In modular combining, one can apply the combining operator to aligned, equal-length parts of operands to get the output corresponding to that part. For example, this is the case with vector (or matrix) addition: to compute any entry in the sum, all that is needed is the corresponding entries in the summands. If the operand is not modular, it is called holistic (e.g., matrix multiplication). We show that in the modular case, using pipelining, a logarithmic factor can be shaved off the running time (more precisely, converted into an additive term). 
We start by defining the cloud intervals of nodes in the wheel settings.

Definition 4.1 The cloud bandwidth of a processing node $i \in V_{p}$ in a given wheel graph is $b_{c}(i) \stackrel{\text { def }}{=}$ $w\left(i, v_{c}\right)$. An interval $[i, i+k] \stackrel{\text { def }}{=}\{i, i+1, \ldots, i+k\} \subseteq V$ is a path of processing nodes in the ring. Given an interval $I=[i, i+k],|I|=k+1$ is its size, and $k$ is its length. The cloud bandwidth of $I$, denoted $b_{c}(I)$, is the sum of the cloud bandwidth of all nodes in $I: b_{c}(I)=\sum_{i \in I} b_{c}(i)$. The bottleneck bandwidth of $I$, denoted $\phi(I)$, is the smallest bandwidth of a link in the interval: $\phi(I)=$ $\min \{w(i, i+1) \mid i, i+1 \in I\}$. If $|I|=1$, define $\phi(I)=\infty$.

For ease of presentation we consider the "one sided" case in which node $i$ does not use one of its incident ring links. As we shall see, this limitation does not increase the time complexity by more than a constant factor. Hence we consider the case in which node $i$ cannot send messages on its counterclockwise link.

Definition 4.2 Let $i$ be a processing node in the wheel settings. We define the following quantities for clockwise intervals; counterclockwise intervals are defined analogously.

- $k_{c}(i)$ is the length of the smallest interval starting at $i$, for which the product of its size by the total bandwidth to the cloud along the interval exceeds s, i.e.,

$$
k_{c}(i)=\min \left(\{n\} \cup\left\{k \mid(k+1) \cdot b_{c}([i, i+k]) \geq s\right\}\right) .
$$

- $k_{\ell}(i)$ is the length of the smallest clockwise interval starting at node $i$, for which the bandwidth of the clockwise-boundary link bandwidth is smaller than the total cloud bandwidth of the interval, i.e.,

$$
k_{\ell}(i)=\min \left(\{n\} \cup\left\{k \mid w(i+k, i+k+1)<b_{c}([i, i+k])\right\}\right) .
$$

- $k(i)=\min \left\{k_{c}(i), k_{\ell}(i)\right\}$.

- $I_{i}=[i, i+k(i)]$. The interval $I_{i}$ is called the (clockwise) cloud interval of node $i$.

- $Z_{i}=\left|I_{i}\right|+\frac{s}{\phi\left(I_{i}\right)}+\frac{s}{b_{c}\left(I_{i}\right)}$. $Z_{i}$ is the timespan of the (clockwise) cloud interval of $i$.

\subsection{The Complexity of $\mathrm{cW}$ and $\mathrm{cR}$}

Theorem 4.1 Given the cloud interval $I_{i}$ of node i, Alg. 2 solves the s-bits $\mathrm{cW}_{i}$ problem in $O\left(Z_{i}\right)$ rounds.

Proof: The BFS tree of the interval would be a simple line graph, that is the whole interval. Note that Step 2 requires $O\left(\left|I_{i}\right|+\frac{s}{\phi\left(I_{i}\right)}\right)$ rounds: there are $s$ bits to send over $\Theta\left(\left|I_{i}\right|\right)$ hops with bottleneck bandwidth $\phi\left(I_{i}\right)$. The rest of the time analysis is the same as in Theorem 3.3.

We have the following immediate consequence.

Theorem 4.2 Let $Z_{i}^{\ell}$ and $Z_{i}^{r}$ denote the timespans of the counterclockwise and the clockwise cloud intervals of $i$, respectively. Then $\mathrm{cW}_{i}$ can be solved in $O\left(\min \left(Z_{i}^{\ell}, Z_{i}^{r}\right)\right)$ rounds.

We now turn to the lower bound.

Theorem 4.3 In the wheel settings, any algorithm for $\mathrm{cW}_{i}$ which does not use link $(i-1, i)$ requires $\Omega\left(Z_{i}\right)$ rounds. 
Proof: We show that each term of $Z_{i}$ is a lower bound on the running time of any algorithm solving $\mathrm{cW}_{i}$.

First, note that any algorithm for $\mathrm{cW}_{i}$ that does not use edge $(i-1, i)$ requires $\Omega\left(k_{c}(i)\right) \geq \Omega(k(i))=$ $\Omega\left(\left|I_{i}\right|\right)$ rounds, due to the exact same arguments as in Lemma 3.5.

Next, we claim that any algorithm for $\mathrm{cW}_{i}$ which does not use edge $(i-1, i)$ requires $\Omega\left(s / \phi\left(I_{i}\right)\right)$ rounds. To see that note first that if $k(i)=0$, then $\phi\left(I_{i}\right)=\infty$ and the claim is trivial. Otherwise, let $(j, j+1) \in E$ be any link in $I_{i}$ with $w(j, j+1)=\phi\left(I_{i}\right)$. Note that $j-i<k(i)$ because $j+1 \in I_{i}$. Consider the total bandwidth of links emanating from the interval $I^{\prime} \stackrel{\text { def }}{=}[i, j]$. Since we assume that the link $(i-1, i)$ is not used, the number of bits that can leave $I^{\prime}$ in $t$ rounds is at most $t \cdot\left(b_{c}\left(I^{\prime}\right)+w(j, j+1)\right)$. Notice that at least $s$ bits have to leave $I^{\prime}$. Observe that $b_{c}\left(I^{\prime}\right) \leq w(j, j+1)$, because otherwise we would have $k_{\ell}(i)=j-i$, contradicting the fact that $k(i)>j-i$. Therefore, any algorithm $A$ that solves $\mathrm{cW}_{i}$ in $t_{A}$ rounds satisfies

$$
s \leq t_{A} \cdot\left(b_{c}\left(I^{\prime}\right)+w(j, j+1)\right) \leq 2 t_{A} \cdot w(j, j+1)=2 t_{A} \cdot \phi\left(I_{i}\right)
$$

and the claim follows.

Finally, we claim that any algorithm $A$ for $\mathrm{cW}_{i}$ which does not use edge $(i-1, i)$ requires $\Omega\left(s / b_{c}\left(I_{i}\right)\right)$ rounds. To see that, recall that $k(i)=\min \left(k_{c}(i), k_{\ell}(i)\right)$. If $k(i)=n$ then $I_{i}$ contains all processor nodes $V_{p}$, and the claim is obvious, as no more than $b_{c}\left(V_{p}\right)$ bits can be written to the cloud in a single round. Otherwise, we consider the two cases: If $k(i)=k_{c}(i)$, then $k(i) \geq s / b_{c}\left(I_{i}\right)-1$ by definition, and we are done since $t_{A}=\Omega(k(i))$. Otherwise, $k(i)=k_{\ell}(i)$. Let us denote $w_{R}=w(i+k(i), i+k(i)+1)$. In this case we have $w_{R}<b_{c}\left(I_{i}\right)$. We count how many bits can leave $I_{i}$. In a single round, at most $b_{c}\left(I_{i}\right)$ bits can leave through the cloud links, and at most $w_{R}$ bits can leave through the local links. Since $A$ solves $\mathrm{cW}_{i}$, we must have $s \leq t_{A} \cdot\left(b_{c}\left(I_{i}\right)+w_{R}\right) \leq 2 t_{A} \cdot b_{c}\left(I_{i}\right)$, and hence $t_{A}=\Omega\left(s / b_{c}\left(I_{i}\right)\right)$.

Theorem 4.4 Let $Z_{i}^{\ell}$ and $Z_{i}^{r}$ denote the timespans of the counterclockwise and the clockwise cloud intervals of $i$, respectively. Then $c W_{i}$ requires $\Omega\left(\min \left(Z_{i}^{\ell}, Z_{i}^{r}\right)\right)$ rounds in the wheel settings.

Proof: Let $T$ be the minimum time required to perform $\mathrm{cW}_{i}$. Due to Lemma 2.3, we know that there is a dynamic flow mapping with time horizon $T$ and flow value $s$ from node $i$ to the cloud. Let $f$ be such a mapping. We assume that no flow is transferred to the source node $i$, as we can modify $f$ so that these flow units would not be sent from $i$ at all until the point where they were previously sent back to $i$. Let $s_{L}$ and $s_{R}$ be the total amount of flow that is transferred on links $(i-1, i)$ and $(i, i+1)$, respectively, and assume w.l.o.g. that $s_{R} \geq s_{L}$. Let $f^{\prime}$ be a new dynamic flow mapping which is the same as $f$, except that no flow is transferred on link $(i-1, i)$. Since $f$ is a valid dynamic flow that transfers all $s$ flow units from $i$ to the cloud, $f^{\prime}$ has flow value at least $s-s_{L}$. Let $A$ be a schedule derived from $f^{\prime}$. The runtime of $A$ is at most $T$ rounds. Let $A^{\prime}$ be a schedule that runs $A$ twice: $A^{\prime}$ would transfer $2\left(s-s_{L}\right)$ bits from node $i$ to the cloud. Since $s \geq s_{R}+s_{L}$, we get that: $2\left(s-s_{L}\right)=2 s-2 s_{L} \geq s+s_{L}+s_{R}-2 s_{L} \geq s$, and thus $A^{\prime}$ solves $\mathrm{cW}_{i}$ without using link $(i-1, i)$. From Theorem 4.3, we get a lower bound for $2 T$ of $\Omega\left(Z_{i}^{r}\right)=\Omega\left(\min \left(Z_{i}^{\ell}, Z_{i}^{r}\right)\right)$.

From Theorem 4.2 and Theorem 4.4 we get the following corollary for the uniform wheel: 
Corollary 4.5 In the uniform wheel topology with cloud bandwidth $b_{c}$ and local link bandwidth $b_{\ell} \geq b_{c}$, $c W$ can be solved in $\Theta\left(\frac{s}{b_{\ell}}+\min \left(\sqrt{\frac{s}{b_{c}}}, \frac{b_{\ell}}{b_{c}}\right)\right)$ rounds. If $b_{\ell}<b_{c}$, the running time is $\Theta\left(s / b_{c}\right)$ rounds.

Proof: If $b_{\ell}<b_{c}, k_{\ell}(i)=0, \phi\left(I_{i}\right)=\infty$ and the result follows. Otherwise, by definition we have $k_{c}(i)=\sqrt{s / b_{c}}-1$ and $k_{\ell}(i)=b_{\ell} / b_{c}-1$, hence $\left|I_{i}\right|=O\left(\min \left(\sqrt{s / b_{c}}, b_{\ell} / b_{c}\right)\right)$. It follows that $b_{c}\left(I_{i}\right)=$ $O\left(\min \left(\sqrt{s \cdot b c}, b_{\ell}\right)\right)$. The result follows by noting that $\phi\left(I_{i}\right)=b_{\ell}$.

For example, for $b_{c}=\sqrt{s}$ and $b_{\ell} \geq s^{3 / 4}$, the running time is $O\left(s^{1 / 4}\right)$.

Remark. Notice that the same upper and lower bounds hold for the $\mathrm{CR}_{i}$ problem as well.

\subsection{Holistic Combining}

We are now ready to adapt Theorem 3.10 to the wheel settings.

Definition 4.3 Given an n-node wheel, let $I_{i}$ be the cloud interval of $i$ with the smaller timespan (cf. Definition 4.2). Define $j_{\max }=\operatorname{argmax}_{i}\left\{\left|I_{i}\right|\right\}, j_{c}=\operatorname{argmin}_{i}\left\{b_{c}\left(I_{i}\right)\right\}$, and $j_{\ell}=\operatorname{argmin}_{i}\left\{\phi\left(I_{i}\right)\right\}$. Finally, define $Z_{\max }=\left|I_{j_{\max }}\right|+\frac{s}{\phi\left(I_{j_{\ell}}\right)}+\frac{s}{b_{c}\left(I_{j_{c}}\right)}$.

In words: $j_{\max }$ is the node with the longest cloud interval, $j_{c}$ is the node whose cloud interval has the least cloud bandwidth, and $j_{\ell}$ is the node whose cloud interval has the narrowest bottleneck.

Let $C$ be the set of all cloud intervals $I_{i}$.

Theorem 4.6 In the wheel settings, cComb can be solved in $O\left(Z_{\max } \log n\right)$ rounds by Alg. 3

The main difference that we can use to our advantage between the wheel case and the general case, is that in the wheel case, for any minimal cover $C^{\prime}, \operatorname{load}\left(C^{\prime}\right) \leq 2$ (see Lemma 4.7). We can therefore use the cover $C$ to build a minimal cover $C^{\prime}$, and then have the multiplexing of Alg. 3 add only a constant factor to the runtime.

We do that by adding another preprocessing stage to Alg. 3, in which we select a cover $C^{\prime} \subseteq C$ such that every node is a member of either one or two intervals of $C^{\prime}$. It is straightforward to find such a cover, say, by a greedy algorithm. Concretely, a cover with a minimal number of intervals is found by the algorithm in [26] (in $O(n \log n)$ sequential time). The covers produced by [26] are sufficient for that matter, as the following lemma states.

Lemma 4.7 Let $C$ be a collection of intervals and denote $U=\bigcup_{I \in C} I$. Let $C^{\prime} \subseteq C$ be a minimalcardinality cover of $U$, and let $\operatorname{load}_{C^{\prime}}(i)=\left|\left\{I \in C^{\prime}: I \ni i\right\}\right|$. Then for all $i \in U, 1 \leq \operatorname{load}_{C^{\prime}}(i) \leq 2$.

Proof: Clearly $\operatorname{load}_{C^{\prime}}(i) \geq 1$ for all $i \in U$ since $C^{\prime}$ is a cover of $U$. For the upper bound, first note that by the minimality of $\left|C^{\prime}\right|$, there are no intervals $I, I^{\prime} \in C^{\prime}$ such that $I \subseteq I^{\prime}$, because in this case $I$ could have been discarded. This implies that the right-endpoints of intervals in $C^{\prime}$ are all distinct, as well as the left-endpoints. Now, assume for contradiction, that there exist three intervals $I, I^{\prime}, I^{\prime \prime} \in C^{\prime}$ such that $I \cap I^{\prime} \cap I^{\prime \prime} \neq \emptyset$ (i.e., there is at least one node which is a member of all three). Then $I \cup I^{\prime} \cup I^{\prime \prime}$ is a contiguous interval. Let $l=\min \left(I \cup I^{\prime} \cup I^{\prime \prime}\right)$ and $r=\max \left(I \cup I^{\prime} \cup I^{\prime \prime}\right) .{ }^{4}$ By counting, one of the three intervals, say $I$, has no endpoint in $\{l, r\}$. But this means that $I \subseteq I^{\prime} \cup I^{\prime \prime}$, i.e., we can discard $I$, in contradiction to the minimality of $\left|C^{\prime}\right|$.

\footnotetext{
${ }^{4}$ The notation $\max (I)$ for an interval $I$ refers to the clockwise-end node of the interval.
} 
As for the "low levels" of the algorithm, i.e. Steps 3-5, a little different approach is required instead of running Alg. 4, due to the limited local bandwidth. We present the time analysis for these steps with the following lemma:

Lemma 4.8 Steps 3-5 of Alg. 3 terminate in $O\left(\left|I_{j_{\max }}\right|+\log \left|I_{j_{\max }}\right| \cdot \frac{s}{\phi\left(I_{j_{\ell}}\right)}\right)$ rounds in the wheel settings, with $P_{I}$ stored in the rightmost node of $I$ for each interval $I \in C^{\prime}$.

Proof: First, we require that each input $S_{i}$ is associated with a single interval in $C^{\prime}$. To this end, we use the rule that if a node $i$ is a member in two intervals $I$ and $I^{\prime}$, then its input $S_{i}$ is associated with the interval $I$ satisfying $\max (I)<\max \left(I^{\prime}\right)$, and a unit input $\tilde{\mathbf{1}}$ is associated with $i$ in the context of $I^{\prime}$, where $\tilde{\mathbf{1}}$ is the unit (neutral) operand for $\otimes$. Intuitively, this rule means that the overlapping regions in an interval are associated with the "left" (counterclockwise) interval.

Second, we assume that the interval size, and therefore the number of leaves in its computation tree, is a power of 2. Otherwise, let $p=2^{\lceil\log |I|\rceil}$ (i.e., $p$ is $|I|$ rounded up to the next power of 2). When doing tree computation over an interval $I$, we extend it (virtually) to a complete binary tree with $p$ leaves, where the leftmost $p-|I|$ leaves have the unit input $\tilde{\mathbf{1}}$. These leaves are emulated by the leftmost node of $I$ (the emulation is trivial).

Assume now that we wish to compute the combined value of an interval $I$ whose length is a power of 2 . Let $S_{i}^{j}$ denote the product of $S_{i}, \ldots, S_{j}$. Computation of the combined value of interval $I$ whose length is a power of 2 proceeds in stages, where each stage $\ell$ computes all level- $\ell$ products in parallel. The algorithm maintains the invariant that after $S_{i}^{j}$ is computed, it is stored in node $j$ (by definition, for all $0 \leq i<n, S_{i}^{i}$ is initially stored at node $i$ ). The computation of a stage is performed as follows.

Let $S_{i}^{j}=S_{i}^{k} \otimes S_{k+1}^{j}$ be a product we wish to compute at level $\ell$, and let $S_{i}^{k}, S_{k+1}^{j}$ be the values held by its children. Note that $k+1-i=j-k=2^{\ell-1}$. The algorithm forwards $S_{i}^{k}$ from node $k$ to node $j$, which multiplies it by (the locally stored) $S_{k+1}^{j}$, thus computing $S_{i}^{j}$, which is stored in node $j$ for the next level. This way, the number of communication rounds is just the time required to forward $s$ bits from $k$ to $j$. Using pipelining, this is done in $j-k+\frac{s}{\phi([k, j])}=2^{\ell-1}+\frac{s}{\phi([k, j])} \leq 2^{\ell-1}+\frac{s}{\phi(I)}$ rounds, and hence the total time required to compute the product of all inputs of any interval $I$ is at most

$$
\sum_{\ell=1}^{\lceil\log |I|\rceil}\left(2^{\ell-1}+\frac{s}{\phi(I)}\right) \leq 2\left(|I|+\log |I| \cdot \frac{s}{\phi(I)}\right) .
$$

Now back to the proof of Lemma 4.8. Correctness is obvious. As for the complexity analysis: By Eq. 9, every interval $B \in C$ completes line 4 in at most $O\left(\left|I_{j_{\max }}\right|+\log \left|I_{j_{\max }}\right| \cdot \frac{s}{\phi\left(I_{j_{\ell}}\right)}\right)$ rounds. Multiplying this bound by $\operatorname{load}(C) \leq 2$ due to the multiplexing, we conclude that the loop of line 3 completes after $O\left(\left|I_{j_{\max }}\right|+\log \left|I_{j_{\max }}\right| \cdot \frac{s}{\phi\left(I_{j_{\ell}}\right)}\right)$ rounds.

Lemma 4.9 In the wheel settings, Step 6 of Alg. 3 terminates in $O\left(Z_{\max } \cdot \log n\right)$ rounds.

Proof: The usage of Alg. 5 remains as it were in the fat-links case. By Lemma 3.14, Step 6 requires $O\left(Z_{\max }\left(C^{\prime}\right) \cdot \operatorname{load}\left(C^{\prime}\right) \cdot \log \left|C^{\prime}\right|\right)$ rounds. Noting that $\left|C^{\prime}\right| \leq|C| \leq n$ and that all intervals in $C^{\prime}$ are cloud intervals, we get an upper bound of $O\left(Z_{\max } \cdot 2 \cdot \log n\right)=O\left(Z_{\max } \cdot \log n\right)$ rounds.

The proof of Theorem 4.6 is completed as follows. The correctness of the algorithm is derived from the general case (Theorem 3.10). As for the time analysis: The "low levels" of the algorithm 
(Steps 3-5) require $O\left(Z_{\max } \log n\right)$ according to Lemma 4.8, noting that $\left|I_{j_{\max }}\right| \leq n$ and that $Z_{\max }=$ $\left|I_{j_{\max }}\right|+\frac{s}{\phi\left(I_{j_{\ell}}\right)}+\frac{s}{b_{c}\left(I_{j_{c}}\right)}$ by definition. The "high level" of the algorithm (Step 6) requires $O\left(Z_{\max } \cdot \log n\right)$ according to Lemma 4.9 .

All in all, the algorithm terminates in $O\left(Z_{\max } \cdot \log n\right)$ rounds.

- Remark: Interval containing node 0 . In the case that $\otimes$ is not commutative, the cover $C^{\prime}$ may need to be patched: In this case, we require that the interval that contains node 0 does not contain node $n-1$. If this is not the case after computing the cover, let $I \in C^{\prime}$ be an interval that contains both. Partition $I$ into two subintervals $I=I_{L} \cup I_{R}$, where $I_{L}$ is the part that ends with node $n-1$, and $I_{R}$ is the part that starts with node $0 . I_{L}$ and $I_{R}$ replace $I$ in the cover $C^{\prime}$ for all purposes, except work: any work assigned to either $I_{L}$ or $I_{R}$ will be executed by all nodes of $I$. This may incur an additional constant slowdown due to multiplexing.

We finish with the lower bound.

Theorem 4.10 Any algorithm for cComb in the wheel topology requires $\Omega\left(Z_{\max }\right)$ rounds.

Proof: Same as in Theorem 3.17, $\Omega\left(Z_{i}\right)$ is a lower bound for every node $i$, by reduction from $\mathrm{cW}_{i}$. Recall that $Z_{i}=\left|I_{i}\right|+\frac{s}{\phi\left(I_{i}\right)}+\frac{s}{b_{c}\left(I_{i}\right)}$. For index $j_{\max }$ we get a lower bound of $\Omega\left(Z_{j_{\max }}\right) \in \Omega\left(\left|I_{j_{\max }}\right|\right)$. For index $j_{\ell}$ we get a lower bound of $\Omega\left(Z_{j_{N}}\right) \in \Omega\left(s / \phi\left(I_{j_{\ell}}\right)\right)$. For index $j_{c}$ we get a lower bound of $\Omega\left(Z_{j_{C}}\right) \in \Omega\left(s / b_{c}\left(I_{j_{c}}\right)\right)$. Summing them all up, gives the desired lower bound.

Remark. We note that similar upper and lower bounds of $O\left(Z_{\max }\right)$ and $\Omega\left(Z_{\max }\right)$ hold for the cCast problem, similarly to the proofs in Sec. 3.2.

\subsection{Modular Combining}

Intuitively, an operator is a modular combining operator if it can be applied to aligned pieces of the operands and obtain the corresponding piece of the result. For example, bit-wise operations are modular, as is vector addition. Formally, we have the following.

Definition 4.4 An operator $\otimes:\{0,1\}^{s} \times\{0,1\}^{s} \rightarrow\{0,1\}^{s}$ is called modular if there exists a partition $s=g_{1}+g_{2}+\cdots+g_{K}$, and $K$ operators $\otimes_{i}:\{0,1\}^{g_{i}} \times\{0,1\}^{g_{i}} \rightarrow\{0,1\}^{g_{i}}$ such that for any $x, y \in$ $\{0,1\}^{s}, x \otimes y$ is equal to the concatenation $\left(x_{1} \otimes_{1} y_{1}\right)\left(x_{2} \otimes_{2} y_{2}\right) \cdots\left(x_{K} \otimes_{K} y_{K}\right)$ with $x=x_{1} x_{2} \cdots x_{K}$, $y=y_{1} y_{2} \cdots y_{K}$ and $\left|x_{i}\right|=\left|y_{i}\right|=g_{i}$ for every $i$. The grain size of $\otimes$ in the given partition is $\max \left\{g_{i} \mid 1 \leq i \leq K\right\}$, and the grain size of $\otimes$ is the minimal grain size of $\otimes$ over all partitions.

Note that any $s$-bits binary operator is trivially modular with grain size at most $s$. However, small grain size facilitates parallelism and pipelining by applying the operation on small parts of the operands independently. Note also that the grain size depends on the way we break down the operands; the best breakdown is the one that minimizes the grain size. Given such a breakdown, in case that all (cloud and local) links have bandwidth at least the grain size, we can use pipelining to convert the logarithmic factor of Theorem 4.6 to an additive term, as the following theorem states.

Theorem 4.11 Let $G=(V, E)$ be a graph of the wheel topology in the CWC model. Suppose that $\otimes$ is modular with grain size $g$, and that $w(e) \geq g$ for every link $e \in E$. Then cComb can be solved in $O\left(Z_{\max }+\log n\right)$ rounds in the wheel settings. 
Similarly to Cor. 4.5, we can get get result for the uniform case.

Corollary 4.12 In the uniform wheel topology with cloud bandwidth $b_{c}$ and local link bandwidth $b_{\ell}$ s.t. $b_{\ell} \geq b_{c}, c$ Comb with an operation of grain size $g \leq b_{c}$ can be executed in $O\left(\frac{s}{b_{\ell}}+\min \left(\sqrt{\frac{s}{b_{c}}}, \frac{b_{\ell}}{b_{c}}\right)+\log n\right)$ rounds.

Proof of Theorem 4.16: From a high level perspective, modular combining is the same as holistic combining, as presented in Theorem 4.6 and Alg. 3. All of the preprocessing steps remain unchanged. The difference is in the implementation of the low level steps (3-5) and the high level step (6).

For a low level step, we note that the computation tree of Lemma 4.8 is no longer necessary; thanks to the modularity of operator $\otimes$, a simple one-pass procedure suffices. For interval $I=[\ell, r]$, the algorithm forwards $S_{\ell}$ from node $\ell$ through the local links all the way to node $r$, and every node along the way combines the grains it receives with its local corresponding grain, and forwards the result grain as soon as it is computed.

More formally, let $i \neq \ell$ be some node in $I$. Let $x=S_{\ell}^{i-1}$ be the operand that $i$ should receive from node $i-1$, and let $y=S_{i}$. We consider the partition of $x$ and $y$ according to the grains of $\otimes$ as described in Definition 4.4. For every grain $x_{j}$ that $i$ fully receives, it calculates locally the product $x_{j} \otimes_{j} y_{j}$ and proceeds to send it immediately to node $i+1$.

Lemma 4.13 Let $\otimes$ be a modular operator with grain size $g$ as in Definition 4.4, and assume that $w(e) \geq g$ for every link $e \in E$. Using pipelining, line 4 of Alg. 3 can be computed in an interval $I$ in $O\left(\frac{s}{\phi(I)}+|I|\right)$ rounds.

Proof: The number of messages required to send all $s$ bits from a node $j$ to node $j+1$ using only local links is at least $\lceil s / \phi(I)\rceil$. An intermediate node can compute the result of a grain and start forwarding it to the next node after $O(1)$ steps at most, since a complete grain is needed. (either a grain was sent in its entirety, or a part of it was and the rest can be sent in the next round.) The lemma follows.

Corollary 4.14 In the settings of Lemma 4.13, line 4 of Alg. 3 can be computed in $O\left(\frac{s}{\phi\left(I_{j_{\ell}}\right)}+\left|I_{j_{\max }}\right|\right) \leq$ $O\left(Z_{\max }\right)$ rounds.

The pipelining strategy applies also to the tree nodes computed in line 6 of Alg. 3. Specifically, we need to change Alg. 5 to work grain-by-grain. Note that we assume the constant-factor multiplexing of Step 2 of Alg. 3, and thus describe the algorithm as if all intervals are operating in complete parallelism. In addition, we assume the bandwidth of all links to be a multiple of $g$ (note that this assumption may only incur a constant factor slowdown, as a link $e$ passing a message of size $w(e)$ can be simulated with 2 rounds of passing messages of sizes $\lfloor w(e) / g\rfloor \cdot g$ and $(w(e) \bmod g)$ since $w(e) \geq g)$.

Suppose that interval $I$ is in charge of filling in the value of a tree node $y$ with children $y_{\ell}$ and $y_{r}$. We assume that the order of reading and writing a string $S$ in $\mathrm{cR}$ and $\mathrm{cW}$ is sequential, i.e., the first bits of $S$ are retrieved (or written, resp.) first. We run, in parallel, cR on $y_{\ell}$ and $y_{r}$, and we also run $\mathrm{cW}$ of $y$ in parallel to them. We can implement this parallelization using multiplexing of the nodes of $I$, while maintaining the same round complexity: the same operations that make up the $\mathrm{cR}$ and cW actions would still occur, it is just the order of the schedule that is tweaked. As soon as a 
grain of $y_{\ell}$ and the corresponding grain of $y_{r}$ are available, the node of $I$ that read them from the cloud computes the corresponding grain of the result and writes it using $\mathrm{cW}$. This way, computing a grain at a tree-node requires $O(1)$ rounds. Hence the first grain is computed at the root after $O(\log n)$ rounds. $\mathrm{cW}$ requires $O\left(Z_{\max }\right)$ rounds, and hence the last grain is written $O\left(Z_{\max }\right)$ rounds later. Thus we obtain the following result.

Lemma 4.15 Let $\otimes$ be a modular operator with grain size $g$ as in Definition 4.4, and assume that $w(e) \geq g$ for every link $e \in E$. Using pipelining, line 6 of Alg. 3 can be computed in $O\left(Z_{\max }+\log n\right)$ rounds.

Theorem 4.16 follows from Cor. 4.14 and Lemma 4.15.

Theorem 4.16 Suppose $\otimes$ is modular with grain size $g$, and that $w(e) \geq g$ for every link $e \in E$. Then cComb can be solved in $O\left(Z_{\max }+\log n\right)$ rounds, where $Z_{\max }=\max _{i \in V_{p}}\left\{\left|I_{i}\right|\right\}+\frac{s}{\min _{i \in V_{p}}\left\{b_{c}\left(I_{i}\right)\right\}}+$ $\frac{s}{\min _{i \in V_{p}}\left\{\phi\left(I_{i}\right)\right\}}$.

\section{CWC Applications}

In this section we briefly explore some of the possible applications of the results shown in this paper to two slightly more involved applications, namely Federated Learning (Sec. 5.1) and File Deduplication (Sec. 5.2).

\subsection{Federated Learning in CWC}

Federated Learning (FL) $[31,11]$ is a distributed Machine Learning training algorithm, by which an ML model for some concept is acquired. The idea is to train over a huge data set that is distributed across many devices such as mobile phones and user PCs, without requiring the edge devices to explicitly exchange their data. Thus it gives the end devices some sense of privacy and data protection. Examples of such data is personal pictures, medical data, hand-writing or speech recognition, etc.

In [8], a cryptographic protocol for FL is presented, under the assumption that any two users can communicate directly. The protocol of [8] is engineered to be robust against malicious users, and uses cryptographic machinery such as Diffie-Hellman key agreement and threshold secret sharing. We propose a way to do FL using only cloud storage, without requiring an active trusted central server. Here, we describe a simple scheme that is tailored to the fat-links scenario, assuming that users are "honest but curious."

The idea is as follows. Each of the users has a vector of $m$ weights. Weights are represented by non-negative integers from $\{0,1, \ldots, M-1\}$, so that user input is simply a vector in $\left(\mathbb{Z}_{M}\right)^{m}$. Let $\mathbf{x}_{i}$ be the vector of user $i$. The goal of the computation is to compute $\sum_{i=0}^{n-1} \mathbf{x}_{i}$ (using addition over $\mathbb{Z}_{M}$ ) and store the result in the cloud. We assume that $M$ is large enough so that no coordinate in the vector-sum exceeds $M$, i.e., that $\sum_{i=0}^{n-1} \mathbf{x}_{i}=\left(\sum_{i=0}^{n-1} \mathbf{x}_{i} \bmod M\right)$.

To compute this sum securely, we use basic multi-party computation in the CWC model. Specifically, each user $i$ chooses a private random vector $\mathbf{z}_{i, j} \in\left(\mathbb{Z}_{M}\right)^{m}$ uniformly, for each of her neighbors 
$j$, and sends $\mathbf{z}_{i, j}$ to user $j$. Then each user $i$ computes $\mathbf{y}_{i}=\mathbf{x}_{i}-\sum_{(i, j) \in E} \mathbf{z}_{i, j}+\sum_{(j, i) \in E} \mathbf{z}_{j, i}$, where addition is modulo $M$. Clearly, $\mathbf{y}_{i}$ is uniformly distributed even if $\mathbf{x}_{i}$ is known. Also note that $\sum_{i} \mathbf{y}_{i}=\sum_{i} \mathbf{x}_{i}$. Therefore all that remains to do is to compute $\sum_{i} \mathbf{y}_{i}$, which can be done by invoking cComb, where the combining operator is vector addition over $\left(\mathbb{Z}_{M}\right)^{m}$. We obtain the following theorem from Theorem 3.15 .

Theorem 5.1 In a fat-links network, an FL iteration with vectors in $\left(\mathbb{Z}_{M}\right)^{m}$ can be computed in $O\left(Z_{\max } \log ^{2} n\right)$ rounds.

Since the grain size of this operation is $O(\log M)$ bits, we can apply the pipelined version (Cor. 4.12) of cComb in case that the underlying topology is a cycle, to obtain the following.

Theorem 5.2 In the uniform n-node wheel, an FL iteration with vectors in $\left(\mathbb{Z}_{M}\right)^{m}$ can be computed in $O\left(\sqrt{(m \log M) / b_{c}}+\log n\right)$ rounds, assuming that $b_{c} m \log M \leq b_{\ell}^{2}$ and $b_{c} \geq \log M$.

Proof: Using the notation of Sec. 3 and Sec. 4, the assumption implies that $s=m \log M$, and $Z_{\max }=O\left(\sqrt{s / b_{c}}\right)=O\left(\sqrt{(m \log M) / b_{c}}\right)$. The result follows from Theorem 4.16 and Cor. 4.12.

\subsection{File Deduplication With the Cloud}

Deduplication, or Single-Instance-Storage (SIS), is a central problem for storage systems (see, e.g., $[17,32,7])$. Grossly simplifying, the motivation is the following: Many of the files (or file parts) in a storage system may be unknowingly replicated. The general goal of deduplication (usually dubbed dedup) is to identify such replications and possibly discard redundant copies. Many cloud storage systems use a dedup mechanism internally to save space. Here we show how the processing nodes can cooperate to carry out dedup without active help from the cloud, when the files are stored locally at the nodes (cf. serverless SIS [13]). We ignore privacy and security concerns here.

We consider the following setting. Each node $i$ has a set of local files $F_{i}$ with their hash values, and the goal is to identify, for each unique file $f \in \bigcup_{i} F_{i}$, a single owner user $u(f)$. (Once the operation is done, users may delete any file they do not own.)

This is easily done with the help of cComb as follows. Let $h$ be a hash function. For file $f$ and processing node $i$, call the pair $(h(f), i)$ a tagged hash. The set $S_{i}=\left\{(h(f), i) \mid f \in F_{i}\right\}$ of tagged hashes of $F_{i}$ is the input of node $i$. Define the operator $\widetilde{\cup}$ that takes two sets $S_{i}$ and $S_{j}$ of tagged hashes, and returns a set of tagged hashes without duplicate hash values, i.e., if $(x, i)$ and $(x, j)$ are both in the union $S_{i} \cup S_{j}$, then only $(x, \min (i, j))$ will be in $S_{i} \widetilde{\cup} S_{j}$. Clearly $\widetilde{\cup}$ is associative and commutative, has a unit element $(\emptyset)$, and therefore can be used in the cComb algorithm. Note that if the total number of unique files in the system is $m$, then $s=m \cdot(H+\log n)$. Applying cComb with operation $\widetilde{\cup}$ to inputs $S_{i}$, we obtain a set of tagged hashes $S$ for all files in the system, where $(h(f), i) \in S$ means that user $i$ is the owner of file $f$. Then we invoke cCast to disseminate the ownership information to all nodes. Thus dedup can be done in CWC in $O\left(Z_{\max } \log ^{2} n\right)$ rounds. 


\section{Conclusion and Open Problems}

In this paper we have introduced a new model that incorporates cloud storage with a bandwidthconstrained communication network. We have developed a few building blocks in this model, and used these primitives to obtain effective solutions to some real-life distributed applications. There are many possible directions for future work; below, we mention a few.

One interesting direction is to validate the model with simulations and/or implementations of the algorithms, e.g., implementing the federated learning algorithm suggested here.

A few algorithmic question are left open by this paper. For example, can we get good approximation ratio for the problem of combining in a general (directed, capacitated) network? Our results apply to fat links and the wheel topologies.

Another interesting issue is the case of multiple cloud nodes: How can nodes use them effectively, e.g., in combining? Possibly in this case one should also be concerned with privacy considerations.

Finally, fault tolerance: Practically, clouds are considered highly reliable. How should we exploit this fact to build more robust systems? and on the other hand, how can we build systems that can cope with varying cloud latency? 


\section{References}

[1] Micah Adler, Phil B. Gibbons, Yossi Matias, and Vijaya Ramachandran. Modeling parallel bandwidth: Local versus global restrictions. Algorithmica, 24:381-404, July 1999.

[2] Yehuda Afek, Gad M. Landau, Baruch Schieber, and Moti Yung. The power of multimedia: Combining point-to point and multi-access networks. In Danny Dolev, editor, Proc. 7th Ann. ACM Symp. on Principles of Distributed Computing, 1988, pages 90-104. ACM, 1988.

[3] Hagit Attiya and Jennifer Welch. Distributed Algorithms. McGraw-Hill Publishing Company, UK, 1998.

[4] John Augustine, Kristian Hinnenthal, Fabian Kuhn, Christian Scheideler, and Philipp Schneider. Shortest paths in a hybrid network model. In Proceedings of the 2020 ACM-SIAM Symposium on Discrete Algorithms, pages 1280-1299, 2020.

[5] Baruch Awerbuch and David Peleg. Sparse partitions (extended abstract). In 31st Annual Symposium on Foundations of Computer Science, St. Louis, Missouri, USA, October 22-24, 1990, Volume II, pages 503-513. IEEE Computer Society, 1990.

[6] Nadine Baumann and Martin Skutella. Solving evacuation problems efficiently-earliest arrival flows with multiple sources. In 2006 47th Annual IEEE Symposium on Foundations of Computer Science (FOCS'06), pages 399-410, 2006.

[7] Bill Bolosky, Scott Corbin, David Goebel, and John (JD) Douceur. Single instance storage in windows 2000. In Proceedings of 4 th USENIX Windows Systems Symposium. USENIX, January 2000. link.

[8] Keith Bonawitz, Vladimir Ivanov, Ben Kreuter, Antonio Marcedone, H. Brendan McMahan, Sarvar Patel, Daniel Ramage, Aaron Segal, and Karn Seth. Practical secure aggregation for privacy-preserving machine learning. In Proceedings of the 2017 ACM SIGSAC Conference on Computer and Communications Security, CCS '17, page 1175-1191, New York, NY, USA, 2017. Association for Computing Machinery.

[9] Rainer E. Burkard, Karin Dlaska, and Bettina Klinz. The quickest flow problem. ZOR - Methods and Models of Operations Research, 37:31-58, 1993.

[10] Keren Censor-Hillel, Dean Leitersdorf, and Volodymyr Polosukhin. Distance computations in the hybrid network model via oracle simulations. Technical Report 2010.13831[cs.DC], arXiv, 2020.

[11] Yong Cheng, Yang Liu, Tianjian Chen, and Qiang Yang. Federated learning for privacy-preserving ai. Commun. ACM, 63(12):33-36, November 2020.

[12] David Culler, Richard Karp, David Patterson, Abhijit Sahay, Klaus Erik Schauser, Eunice Santos, Ramesh Subramonian, and Thorsten von Eicken. LogP: Towards a realistic model of parallel computation. SIGPLAN Not., 28(7):1-12, July 1993. 
[13] J. R. Douceur, A. Adya, W. J. Bolosky, P. Simon, and M. Theimer. Reclaiming space from duplicate files in a serverless distributed file system. In Proceedings 22nd International Conference on Distributed Computing Systems, pages 617-624, 2002.

[14] Dropbox. Prospectus, 2018. Filing to US Securities and Exchange Commission. https://www.sec.gov/Archives/edgar/data/1467623/000119312518055809/d451946ds1.htm.

[15] Lisa Fleischer and Martin Skutella. Quickest flows over time. SIAM J. Comput., 36(6):1600-1630, February 2007.

[16] Pierre Fraigniaud. Distributed computational complexities: Are you Volvo-addicted or NASCARobsessed? In Proc. 30th Ann. ACM Symp. on Principles of Distributed Computing, PODC '10, pages 171-172, New York, NY, USA, 2010. Association for Computing Machinery.

[17] Larry Freeman. Looking beyond the hype: Evaluating data deduplication solutions. http://www-download.netapp.com/edm/TT/docs/Looking_beyond_hype_Dedupe.pdf, September 2007. Network Appliance, Inc.

[18] Roy Friedman, Gabriel Kliot, and Alex Kogan. Hybrid distributed consensus. In Roberto Baldoni, Nicolas Nisse, and Maarten van Steen, editors, Principles of Distributed Systems - 17th International Conference, OPODIS 2013, Nice, France, December 16-18, 2013. Proceedings, volume 8304 of Lecture Notes in Computer Science, pages 145-159. Springer, 2013.

[19] Phillip B. Gibbons, Yossi Matias, and Vijaya Ramachandran. Can shared-memory model serve as a bridging model for parallel computation? In Proceedings of the Ninth Annual ACM Symposium on Parallel Algorithms and Architectures, SPAA '97, page 72-83, New York, NY, USA, 1997. Association for Computing Machinery.

[20] James W. Hegeman and Sriram V. Pemmaraju. Lessons from the congested clique applied to MapReduce. Theor. Comput. Sci., 608(P3):268-281, December 2015.

[21] B. Hoppe and É. Tardos. Polynomial time algorithms for some evacuation problems. In SODA '94, 1994.

[22] Howard Karloff, Siddharth Suri, and Sergei Vassilvitskii. A model of computation for MapReduce. In Proceedings of the Twenty-First Annual ACM-SIAM Symposium on Discrete Algorithms, SODA '10, pages 938-948, USA, 2010. Society for Industrial and Applied Mathematics.

[23] Fabian Kuhn and Philipp Schneider. Computing shortest paths and diameter in the hybrid network model. In Yuval Emek and Christian Cachin, editors, PODC '20: ACM Symposium on Principles of Distributed Computing, Virtual Event, Italy, August 3-7, 2020, pages 109-118. ACM, 2020.

[24] Richard E. Ladner and Michael J. Fischer. Parallel prefix computation. J. ACM, 27(4):831-838, October 1980.

[25] Frederic Lardinois. Google drive will hit a billion users this week. TechCrunch, July 2018. https://techcrunch.com/2018/07/25/google-drive-will-hit-a-billion-users-this-week. 
[26] C.C. Lee and D.T. Lee. On a circle-cover minimization problem. Information Processing Letters, 18(2):109 - 115, 1984.

[27] Ming Li and Paul Vitányi. An Introduction to Kolmogorov Complexity and its Applications. Springer International, Switzerland, 4th edition, 2019.

[28] Nathan Linial. Locality in distributed graph algorithms. SIAM J. Comput., 21:193-201, 1992.

[29] Zvi Lotker, Boaz Patt-Shamir, Elan Pavlov, and David Peleg. Minimum-weight spanning tree construction in $O(\log \log (n))$ communication rounds. SIAM J. Comput., 35(1):120-131, 2005.

[30] Yishay Mansour, Noam Nisan, and Uzi Vishkin. Trade-offs between communication throughput and parallel time. J. Complex., 15(1):148-166, 1999.

[31] Brendan McMahan, Eider Moore, Daniel Ramage, Seth Hampson, and Blaise Agüera y Arcas. Communication-efficient learning of deep networks from decentralized data. In Proc. 20th International Conference on Artificial Intelligence and Statistics, (AISTATS), pages 1273-1282, 2017.

[32] Dutch T. Meyer and William J. Bolosky. A study of practical deduplication. ACM Trans. Storage, 7(4), February 2012.

[33] David Peleg. Distributed Computing: A Locality-Sensitive Approach. Society for Industrial and Applied Mathematics, Philadelphia, PA, USA, 2000.

[34] Martin Skutella. An introduction to network flows over time. In William J. Cook, László Lovász, and Jens Vygen, editors, Research Trends in Combinatorial Optimization. Springer, Berlin, Heidelberg, 2009.

[35] Leslie G. Valiant. A bridging model for parallel computation. Commun. ACM, 33(8):103-111, August 1990. 